\title{
Simulating Fluid Flows in Micro and Nano Devices: The Challenge of Non-Equilibrium Behaviour
}

\author{
Jason M Reese ${ }^{*}$ and Yonghao Zhang \\ Department of Mechanical Engineering, University of Strathclyde, Glasgow G1 1XJ, UK
}

\begin{abstract}
We review some recent developments in the modelling of non-equilibrium (rarefied) gas flows at the micro- and nano-scale, concentrating on two different but promising approaches: extended hydrodynamic models, and lattice Boltzmann methods.

Following a brief exposition of the challenges that non-equilibrium poses in micro- and nano-scale gas flows, we turn first to extended hydrodynamics, outlining the effective abandonment of Burnett-type models in favour of high-order regularised moment equations. We show that the latter models, with properly-constituted boundary conditions, can capture critical non-equilibrium flow phenomena quite well. We then review the boundary conditions required if the conventional Navier-Stokes-Fourier (NSF) fluid dynamic model is applied the micro scale, describing how $2^{\text {nd }}$-order Maxwell-type conditions can be used to compensate for some of the non-equilibrium flow behaviour near solid surfaces. While extended hydrodynamics is not yet widely-used for real flow problems because of its inherent complexity, we finish this section with an outline of recent 'phenomenological extended hydrodynamics' (PEH) techniques - essentially the NSF equations scaled to incorporate nonequilibrium behaviour close to solid surfaces - which offer promise as engineering models.

Understanding non-equilibrium within the lattice Boltzmann (LB) framework is not as advanced as in the hydrodynamic framework, although LB can borrow some of the techniques which are being developed in the latter - in particular, the near-wall scaling of certain fluid properties that has proven effective in PEH. We describe how, with this modification, the standard $2^{\text {nd }}$-order LB method is showing promise in predicting some rarefaction phenomena, indicating that instead of developing higher-order off-lattice LB methods with a large number of discrete velocities, a simplified high-order LB method with near-wall scaling may prove to be just as effective as a simulation tool.
\end{abstract}

Keywords: microfluidics, nanofluidics, rarefied gas dynamics, non-equilibrium fluid dynamics, slip flow, Knudsen layer, micro- and nano-scale flows, extended hydorodynamics, lattice Boltzmann

\section{Introduction}

The set of Navier-Stokes-Fourier (NSF) equations, with no-velocity-slip and no-temperature-jump conditions at solid bounding surfaces, is the traditional model for the near-equilibrium transfer of heat and momentum in fluid flows. While it has proven successful for flows ranging from liquids in capillaries to the atmospheres of planets, remarkably it can be a very poor predictor of micro-scale gas flows and nano-scale liquid flows. This is because some surprising non-equilibrium effects occur in these types of flows that are not obvious under more conventional (macro-scale) circumstances.

\footnotetext{
*Corresponding author; jason.reese@strath.ac.uk
} 
While flow non-equilibrium remained essentially a curiosity from the late $19^{\text {th }}$-century through to the mid $20^{\text {th }}$-century, in order to model and optimise the performance of newly-emerging technologies that manipulate fluids at the smallest scales it is now important to address this weakness in our understanding. The non-intuitive flow physics involved also represents an opportunity to engineer devices with capabilities beyond any currently conceived.

In this paper we describe some of the most recent computational and theoretical tools that engineers and scientists are bringing to bear on this problem. We focus on gas micro flows, because in this case methods grounded in the powerful kinetic theory of gases can often be deployed effectively.

Gas micro- and nano-flow devices are being developed for a broad range of applications, from extracting biological samples to active aerodynamic flow control ${ }^{1,2}$. However, gases confined in smallscale geometries behave differently from their macro-scale flow counterparts. Examples of this behaviour include: increased flowrates caused by velocity slip at the wall and the Knudsen layers ${ }^{3}$, inverted velocity profiles in cylindrical Couette flows ${ }^{4}$, and the 'Knudsen minimum' in channel flowrates ${ }^{5}$. This unique non-equilibrium flow behaviour can also be exploited to make devices with new capabilities, e.g., the Knudsen pump/compressor has no moving parts ${ }^{6}$.

Gas flows in miniaturised devices are often low-speed, low-Reynolds-number, and non-equilibrium (rarefied). The Knudsen number, $K n$, indicates whether a gas is in local thermodynamic equilibrium; in terms of the molecular mean-free-path, $\lambda$, and a characteristic length scale, $L$, of the fluid system:

$$
K n=\frac{\lambda}{L} \cong \frac{\lambda}{Q}\left|\frac{d Q}{d l}\right|,
$$

where / represents a suitable spatial direction and $Q$ is a quantity of interest, such as the gas density, pressure or temperature. When the mean-free-path of the gas molecules approaches the length scale of the flow system, microscopic effects and bulk flow effects become increasingly coupled. Lack of both scale separation and local thermodynamic equilibrium means that the linear constitutive relations for viscous-stress and heat-flux, which underpin the NSF set of equations, are no longer valid.

The NSF equations with no-velocity-slip and no-temperature-jump bounding wall conditions are generally only appropriate when $K n<0.001$, but gas flows in micro- and nano-devices are often in the slip regime $(0.001<K n<0.1)$ or the transition-continuum regime $(0.1<K n<10)$. In these regimes, the gas flows cannot properly be described as "quasi-equilibrium continuum", nor quite as "free molecular". In practice, most devices operate with a range of Knudsen numbers in different parts of the device; this makes it even more difficult to develop a generalised flow model.

Traditionally, research into non-equilibrium gas flows has been for high-speed (usually aerodynamic) applications. Highly accurate methods have been developed for these flows, including directly solving the Boltzmann equation and using the direct simulation Monte Carlo (DSMC) method. However, for low-speed gas flows, accurate solutions from these kinetic methods are often beyond current computer capabilities, certainly for practical device design purposes in 3D; DSMC suffers large statistical scatter, while direct solution of the Boltzmann equation is very computationallydemanding ${ }^{7,8}$. While DSMC, molecular dynamics, and direct kinetic methods are beyond the scope of this review paper - and really require a separate article of their own ${ }^{9}-$ in the absence of experimental micro- or nano-flow data they are often relied on to provide independent benchmark 
data; many of the models outlined below are explicitly validated against DSMC or other numerical/molecular data.

It took some 30 years for computational fluid dynamics (CFD) to develop from an academic research topic into an industrial design tool, but the current rapid development of micro- and nanotechnologies means the need for reliable, accurate and computationally-efficient non-equilibrium flow models is more urgent. Two leading contenders for such models for gas flows are extended hydrodynamics and mesoscopic lattice Boltzmann (LB) methods. Both have the advantage of being more computationally-efficient than molecular-based methods, and both are the focus of much current work on improving their applicability to non-equilibrium flows. In the remainder of this paper we outline the development of these two types of methods and discuss both their current achievements and future challenges.

\section{Hydrodynamic approaches}

\subsection{Extended hydrodynamics}

The conventional description of heat and momentum transport in fluid flows is the NSF model, which is regarded as being accurate only to $1^{\text {st }}$-order in $\mathrm{Kn}$. 'Extended hydrodynamics' refers to transport models whose approximate nature is more suitable for resolving high-Kn phenomena. While these models encompass the NSF equations, they include additional terms or equations that are generally higher-order (and typically non-linear) in the gradients of flow quantities.

Extended hydrodynamics is under active investigation for micro and nano gas flows because it is presumed it should not be much more expensive computationally than the standard NSF equations, so complex simulations in 3D could become numerically much more tractable. Another important advantage is that the extended models reduce to NSF hydrodynamics in regions of low $K n$, so in principle the same set of equations can be applied across an entire mixed-rarefaction flow field.

A survey of the literature indicates there have been more than a dozen different extended hydrodynamic models proposed over the last 20 years or so. The majority of these stem from two alternative approaches to solving the Boltzmann equation for a gas, i.e.:

$$
\frac{\partial f}{\partial t}+\boldsymbol{v} \cdot \frac{\partial f}{\partial \boldsymbol{r}}+\boldsymbol{F} \cdot \frac{\partial f}{\partial \boldsymbol{v}}=C(f),
$$

where $f(\boldsymbol{r}, \boldsymbol{v}, t)$ is the function describing the number density of gas molecules with position $\boldsymbol{r}$ and velocity $\boldsymbol{v}$ at a certain time $t$, under the influence of a body force $\boldsymbol{F}$, and suffering binary collisions described through the production term $C(f)$. Equation (2) is presumed to be a suitable model for the rarefied gas flows that occur in micro and nano systems, so if $f$ can be calculated then the state of the gas in the system is known.

DSMC and other direct methods for solving for the distribution function, $f$, are beyond the scope of this paper, so we focus here on approximate methods. The classical approach to solving equation (2) is the Chapman-Enskog technique, where an approximate solution to the molecular velocity distribution function is proposed as a perturbation series in increasing order in $K n$ around the equilibrium Maxwellian distribution. The presumption is that the more terms that are taken in this series, the better the approximation to the non-equilibrium distribution function. The expressions for the viscous fluid stress and heat-flux that then follow from taking successive terms in this 
approximation are: the Navier-Stokes equations at $1^{\text {st }}$-order, the Burnett equations at $2^{\text {nd }}$-order, and the super-Burnett equations at $3^{\text {rd }}$-order in $\mathrm{Kn}$. Chapman and Cowling ${ }^{10}$ give a good introduction and overview of this highly mathematical topic.

Since the first derivation of the Burnett equations in 1935, ample evidence has mounted of their, and the super-Burnett equations', inability to reproduce non-equilibrium flow phenomena accurately. They cannot reproduce Knudsen layer behaviour in micro-flows ${ }^{11}$, and they also demonstrate pathological instabilities for even simple flows ${ }^{12,13}$. We can say with near-definitiveness that the Burnett and super-Burnett equations are not a promising route for further investigation, even with the ad hoc corrections sometimes proposed to alleviate the problems with them. (Although see section 2.2 below and also the work of Lockerby and Reese ${ }^{14}$ for examples of how the Burnett equations, applied carefully, can provide insight into the flow behaviour.)

A more promising method of solving the Boltzmann equation was proposed by $\mathrm{Grad}^{15}$. He expanded the molecular distribution function up to $3^{\text {rd }}$-order in a series of Hermite tensor polynomials, with variable parameters, around the Maxwellian equilibrium state. What are termed Grad's 13-moment equations are retrieved for 13 dependent variables in the conservation equation set. These are somewhat more complicated than the Burnett and super-Burnett equations, in that they involve a coupling of the viscous stress with the heat-flux.

Struchtrup ${ }^{13}$ and Struchtrup and Torrilhon ${ }^{16,17}$ proposed regularised 13-moment equations (denoted the R13 equations), which are similar to Grad's equations but introduce some higher-order effects through a first-order perturbation of the 13-moment expansion. In this framework, the NavierStokes-Fourier relations are the 'R5 equations' (i.e. a first-order perturbation of the 5-moment, or Euler, equations), so the R13 equations require an additional 8 transport equations to be solved.

This process need not stop at 13 moments and can proceed to arbitrary order; the R20 equations (which follow from a first-order perturbation of the complete $3^{\text {rd }}$-order polynomial set) have been recently derived for Maxwell-molecules and investigated by Mizzi and co-workers ${ }^{18,19}$. Space limitations here preclude detailed description of the derivations of the R13 and R20 models, but these can be found in the relevant literature just cited.

The lengthiness and complexity of the Grad, R13 and R20 governing equation sets, coupled with the difficulty in ascribing much, if any, physical meaning to many of the higher-order moments involved (which are sometimes described as 'fluxes of fluxes'), has meant that progress in their analysis has been slow, and take-up among non-equilibrium fluid dynamicists has been patchy. However, these moment equations do generally display temporal and spatial stability for bulk flows ${ }^{12,13}$, unlike their Burnett counterparts, so at present they are the most promising of the extended hydrodynamic models.

Specifying the additional boundary conditions required for these higher-moment equations remains, however, a critical problem. In the case of the R20 model, most recently Mizzi ${ }^{18}$ has systematically derived boundary conditions that describe the micro-processes in a Maxwell-type phenomenological manner (see also section 2.3). In brief, the bounding wall is assumed non-porous so half-space moments are considered, and the net flux of any moment should then be zero. By applying this balance representation iteratively on all the moments considered, boundary conditions follow that are appropriate for any of the Grad-type models ${ }^{18,19}$. 
We will now show some of the micro flow results that can be obtained using the R20 model with these boundary conditions.

We have already mentioned Knudsen layer flows a couple of times in this paper; from a molecular viewpoint, the Knudsen layer is the region close to a solid surface over which the discontinuity introduced into the distribution function by gas/surface interactions (with a surface usually modelled as a Maxwellian emitter) relaxes back to its bulk flow characteristic. This usually occurs over one or two mean-free-paths. Figure 1 shows schematics and gives further (hydrodynamic) explanation of both the momentum and thermal Knudsen layers that occur generally in flows. While Knudsen layers exist close to surfaces in any gas flow, they only become important at the micro- and nano-scale; in fact, for $K n \geq 0.1$ they dominate the flow behaviour. They represent a critical test case for extended hydrodynamics because hydrodynamic models based on linear departures from local thermodynamic equilibrium, such as the NSF equations, cannot capture the non-linear Knudsen layer behaviour.

Figure 2 shows the velocity profiles in Couette flow (i.e. a constant shear stress problem), for $K n=0.5$, calculated using various hydrodynamic models and compared with benchmark DSMC data ${ }^{20}$. All the solutions capture quite well the amount of velocity slip at the solid walls, but the non-linear behaviour seen in the molecular dynamics DSMC results close to the walls is more difficult to reproduce. The R20 model with its new boundary conditions displays the best agreement with DSMC, but it should be noted that any hydrodynamic model is likely to have difficulty in capturing the flowfield at such a relatively high $K n$.

Figure 3 shows the temperature profiles in a force-driven channel (Poiseuille) flow case, for $K n=0.1$. In a non-equilibrium channel-flow the temperature profile shows a distinct bimodality, which is another good test for any new hydrodynamic model. In this case, again, the R20 model shows the most promising agreement with DSMC (with a hard-sphere molecular model), although it should be said that even this is not strong quantitatively. For interest, included in this figure are results of using the R20 model but with a hard-sphere viscosity/temperature relationship.

Finally, figure 4 demonstrates an interesting phenomenon: reversed heat-fluxes ${ }^{18,21}$. In this case, a 2D cavity has been simulated, with a driven-lid at the top of the cavity moving from left to right in the figure at Mach 0.1; the flow Reynolds number is 1.5. All spatial dimensions have been normalised, but the temperature is reported in degrees $\mathrm{K}$. The R20 hydrodynamic model can capture qualitatively the reversed heat-fluxes predicted by DSMC. This rarefaction behaviour is also reproducible in kinetic theory solutions, but not in the NSF model. Such phenomena may be important in understanding thermal load management and heat dissipation in MEMS and computer CPUs.

\subsection{Boundary conditions}

The problem of properly defining additional boundary conditions for extended hydrodynamics, as outlined in the previous section, means that many micro- and nano-scale gas flow investigations are still conducted within the conventional NSF framework. However, there are still issues surrounding the correct implementation of non-equilibrium boundary conditions. To illustrate, we confine our attention here to models for calculating the slip-velocity to be imposed on hydrodynamic simulations at solid bounding surfaces, although calculation of the temperature-jump at surfaces also requires careful consideration ${ }^{22}$. 
While there has been recent interest in using a Langmuir adsorption model of the gas/surface interaction to retrieve rarefied flow boundary conditions ${ }^{23,24}$, for flows of $K n>0.001$ Maxwell's phenomenological expression ${ }^{25}$ remains the most often-used expression for the tangential slipvelocity, $\boldsymbol{u}_{\text {slip }}$ :

$$
\boldsymbol{u}_{\text {slip }}=-\frac{2-\sigma}{\sigma \mu} \lambda \boldsymbol{\tau}-\frac{3}{4} \frac{\operatorname{Pr}(\gamma-1)}{\not p} \boldsymbol{q},
$$

where $\boldsymbol{\tau}$ is the tangential shear stress and $\boldsymbol{q}$ the tangential heat-flux at the surface, $\sigma$ the momentum accommodation coefficient (ranging from 1 for surfaces that reflect all incident molecules diffusely, to 0 for purely specular reflection), $\mathrm{Pr}$ is the gas Prandtl number, $\mu$ the gas viscosity, $\gamma$ the specific heat ratio, and $p$ the gas pressure.

Because equation (3) requires the viscous-stress and heat-flux, we can choose to use the NSF constitutive expressions for these, as Maxwell himself did. For a flow bounded by planar surfaces (and for a perfect gas), we then obtain the conventional equation for the magnitude of the slipvelocity tangential to the surface, viz.

$$
u_{\text {slip }}=\frac{2-\sigma}{\sigma} \lambda \frac{\partial u_{x}}{\partial n}+\frac{3}{4} \frac{\mu}{\rho T} \frac{\partial T}{\partial x},
$$

where $n$ is the coordinate normal to the surface and $x$ the tangential coordinate, $u_{x}$ is the $x$ component of the gas velocity, and $\rho$ and $T$ are the density and temperature, respectively, of the gas at the wall.

While equation (4) has the advantage of simplicity, it should be remembered that it is incorrect for simulations of gas flows over curved or rotationally-moving surfaces, such as serpentine bends, spheres and cylinders, and other geometries in which the velocity normal to the surface varies in the streamwise direction (such as deflecting flaps). In such cases, using equation (4) instead of equation (3) misses out some important flow physics.

For example, figure 5 shows that the different boundary conditions produce radically different predictions of cylindrical micro Couette flow ${ }^{4}$. In this figure it is the inner cylinder that is rotating, but independent DSMC simulations show that the high gas velocities actually occur at the stationary outer cylinder wall. This curious rarefaction phenomenon is only captured by hydrodynamic models if the full Maxwell slip equation (3) is used, not the conventional slip equation (4). The no-slip fluid dynamic solution and an independent analytical result are also shown on this figure, for comparison. (Note that, for this flow system, differences in the results caused by using different boundary condition models are accentuated by the artificially low momentum accommodation coefficient.)

The conventional slip-velocity expression (4) is $1^{\text {st }}$-order in flow property gradients, but $2^{\text {nd }}$-order slip conditions have been proposed to account for the effect of Knudsen layers. These conditions compensate for the existence of a Knudsen layer through changing the magnitude of the slipvelocity, but they do not model the Knudsen layer structure itself. A $2^{\text {nd }}$-order formulation calculates a different magnitude for the slip-velocity in an attempt to capture the increase in mass flowrates observed at higher $K n$, but it cannot alter the near-wall stress/strain-rate (constitutive) behaviour which characterises the Knudsen layer. In the schematics of Knudsen layers in figure $1,2^{\text {nd }}$-order boundary conditions model the 'fictitious' slip-velocity at the surface, while the linear NSF model is generally retained for the flow away from the surface. 
The general form of these $2^{\text {nd }}$-order conditions is, for planar surfaces,

$$
u_{\text {slip }}=A_{1} \lambda \frac{\partial u_{x}}{\partial n}-A_{2} \lambda^{2} \frac{\partial^{2} u_{x}}{\partial n^{2}}
$$

where we have also now assumed there is no heat flux along the surface. The values to be assigned to the coefficients $A_{1}$ and $A_{2}$ are still the subject of much discussion, and are in any case likely to be geometry-dependent. Table 1 gives a selection of some of the values in the literature, proposed either from theoretical considerations or inferred from experimental observations. Note that a proper comparison of different slip models and their coefficients requires consistency in the expression used to determine $\lambda$, and the values assigned to $\sigma$ (q.v. the discussion in section 3.2 of a similar problem in comparing different lattice Boltzmann results).

While the form of equation (5) has been proposed because researchers wish to account for any unevaluated $2^{\text {nd }}$-order contributions to the slip-velocity, the original Maxwell slip condition (3) was proposed from physical considerations. Inspection of this equation, and its original derivation ${ }^{25}$, shows that there is no a priori restriction on the expressions for the stress or heat-flux that it requires. While Maxwell used the NSF model, we are free to use another constitutive model; Lockerby and co-workers ${ }^{4}$ used the Burnett expression for the viscous-stress to derive a linearised 'Maxwell-Burnett' boundary condition, which is formally $2^{\text {nd }}$-order in space and stable in its solution. Expressing this new boundary condition in the form of equation (5) allows its coefficients $A_{1}$ and $A_{2}$ to be included in Table 1; it can be seen from the table that these coefficients are generally in line with many of the other coefficient values.

While the general problems with the Burnett equations outlined in section 2.1 still stand, the justification for using them in this new $2^{\text {nd }}$-order boundary condition is that they may at least be better approximations than the NSF model for the non-equilibrium stress and heat-flux close to a surface. In support of this, Lockerby and co-workers ${ }^{4}$ showed that the new Maxwell-Burnett boundary condition, applied with a conventional NSF hydrodynamic model for the bulk flow, generates a thermal-stress slip flow (as distinct from a thermal creep flow) when there is a tangential variation in the wall-normal temperature gradient. While this flow behaviour cannot be captured by Maxwell's boundary condition with an NSF model for the shear stress, its existence was first deduced by Sone ${ }^{34}$ from kinetic theory arguments.

The Maxwell-Burnett boundary condition has the advantage of relative simplicity because the Burnett equations provide an explicit expression for the viscous-stress, but in principle an R13 or R20 constitutive model (see section 2.1) in equation (3) would also be possible and may be advantageous.

\subsection{Near-wall constitutive scaling ('phenomenological extended hydrodynamics')}

The lengthy complexity of most extended hydrodynamics models, with their boundary conditions, has led recently to a re-evaluation of modified versions of the NSF equations to be used as 'engineering models'. This has followed from the observation that the NSF equations with slip boundary conditions are a surprisingly reasonable, as well as computationally-efficient, model in many cases. The modifications proposed are to incorporate some of the essential non-equilibrium behaviour near solid surfaces. While some accuracy (relative to extended hydrodynamics proper) is sacrificed in favour of ease of application, a major advantage of this approach to modelling non- 
equilibrium flows is that no additional boundary conditions beyond the slip/jump conditions outlined in section 2.2 are required.

This approach has been termed 'phenomenological extended hydrodynamics' (PEH), and incorporates a constitutive scaling of the NSF equations that depends on distance to the nearby solid surface. The physical basis for this constitutive scaling can be traced to the work of Stops ${ }^{35}$, who demonstrated that the molecular mean-free-path of a gas is foreshortened (in comparison to its bulk flow value) close to an immovable solid surface. As the mean-free-path can be directly related to the viscosity and thermal conductivity of the gas, the fluid properties and behaviour of the gas some one to two mean-free-paths from a surface (i.e. in the Knudsen layer) are therefore expected to be different to those in the bulk flow.

Guo and co-workers ${ }^{36}$ developed a numerical solution method for Stops' original expression for the mean-free-path which, together with $2^{\text {nd }}$-order slip boundary conditions, produced good predictions for phenomena such as the Knudsen minimum in Poiseuille flow (see figure 6). Fichman and Hetsroni $^{37}$, and $\mathrm{Li}$ and colleagues $^{38}$, used independent mean-free-path arguments to propose that both the viscosity and the thermal conductivity of the gas fall monotonically and uniformly to half their bulk flow values across the region of a couple of mean-free-paths up to a surface. Analogous work includes viscosity-modification models for thin-film lubrication flows by Sun and co-workers ${ }^{39}$, and Schrag and Wachutka ${ }^{40}$ (the latter building on a previous model by Veijola and others ${ }^{41}$ ).

Some of the most recent work in this field focuses on developing relatively simple analytical forms of the mean-free-path variation close to surfaces, from which non-linear constitutive relations can be straightforwardly derived. These modified NSF relations incorporate wall-distance scaling functions derived from planar kinetic theoretical results ${ }^{12,22,42,43,44,45}$. For example, for isothermal cases this scaling acts to change the form of the 3D low-speed steady-state incompressible NSF momentum equation to:

$$
\nabla p=2 \mu \nabla \cdot(\Phi \overline{\nabla U})=\mu \Phi \nabla^{2} U+2 \mu \nabla \Phi \cdot \overline{\nabla U},
$$

where $U$ is the gas flow velocity and $\overline{\nabla U}=\left[\nabla U+(\nabla U)^{T}\right] / 2$. The near-wall scaling function, $\Phi$, in this equation is given by

$$
\Phi=\left[1+\Psi_{1}(\hat{n})+K \Psi_{2}(\hat{n})\right]^{-1},
$$

where $\hat{n}$ is the perpendicular distance (nondimensionalised with $\lambda$ ) from the nearest wall surface ${ }^{12}$.

Lilley and Sader ${ }^{46,47}$, drawing on Boltzmann theoretical solutions for hard-sphere molecules, propose a power-law structure to the inner part of the momentum Knudsen layer, with the consequence that the viscosity of the gas closest to the solid surface is effectively zero. The PEH model of Lockerby and Reese $^{12}$ adopts a similar power-law structure, viz. the test functions $\Psi_{i}$ in equation (7) are of the form:

$$
\Psi_{i}(\hat{n})=a_{i} \hat{n}^{b_{i}} \exp \left\{c_{i} \hat{n}\right\}
$$

and $a_{i}, b_{i}$ and $c_{i}$ are coefficients that are determined once by optimisation from benchmark kinetic theory solutions to be:

$$
\begin{array}{lll}
a_{1}=0.1859, & b_{1}=-0.4640, & c_{1}=-0.7902 ; \\
a_{2}=0.4205, & b_{2}=-0.3518, & c_{2}=-0.4521 .
\end{array}
$$

The flow-dependent variable, $K$, in equation (7) is in essence a form of local Knudsen number, and is introduced to provide a $2^{\text {nd }}$-order component to the constitutive scaling: 


$$
K=\frac{1}{\tau} \frac{\mathrm{d} \tau}{\mathrm{d} \hat{n}}
$$

where the shear stress $\tau=\boldsymbol{i}_{\hat{x}} \cdot\left(\boldsymbol{i}_{\hat{n}} \cdot \Pi\right)$, $\Pi$ is the viscous stress tensor, $\boldsymbol{i}_{\hat{n}}$ a unit vector in the wallnormal direction, and $\boldsymbol{i}_{\hat{x}}$ a unit vector perpendicular to $\boldsymbol{i}_{\hat{n}}$ in a direction that gives maximum $\tau$. This $\mathrm{PEH}$ model as a whole can indirectly (although will not necessarily) affect the shear stress field, which in turn will alter $K$, producing a weak coupling effect.

PEH equations are generally easy to incorporate into current CFD codes and, despite their simplicity, their solutions are often in excellent agreement with a range of planar gas flow data (including Couette and Poiseuille flows). Perhaps surprisingly, given that the coefficients (9) for the testfunctions are derived from planar kinetic theory results, Lockerby and Reese's model also shows good agreement in non-planar gas flow problems, such as the flow around a micro-sphere (see figure 7). It even shows moderate success when applied to high-speed thermal rarefied gas flows, e.g. in hypersonic aerothermodynamics ${ }^{48}$, despite having mainly been derived for isothermal micro flows. In the form of effective mean-free-path models, near-wall scaling is even being introduced into lattice Boltzmann methods (see section 3.3). This seems to indicate a useful generality to the PEH technique which is worth exploring in the future.

While PEH offers some exciting new possibilities, and shows an ability to capture some essential features of non-equilibrium flows at the micro scale, it will never be as accurate - in terms of recovering the detailed flow behaviour - as a rational extended hydrodynamics model based on the fundamental physics. For example, the simple wall-distance scaling models described above do not currently capture the bimodal temperature profile that is a prominent feature of force-driven Poiseuille flow (as seen in figure 3). However, our view is that PEH fits into a hierarchy or 'spectrum' of simulation tools which the scientist or engineer can deploy on micro- or nano-flow problems, depending on the accuracy required of the result and the time allowed to achieve it.

At one end of this 'spectrum' lie molecular techniques (such as DSMC), which produce very accurate results but are at present computationally intractable for 3D flow systems. At the other end of the spectrum lies the conventional NSF model, whose numerical solution in 3D for near-equilibrium engineering problems is now commonplace. Extended hydrodynamics lies between these two limits, with the moment equations of section 2.1 (with their inherent accuracy, but complexity and difficulty in evaluation) lying towards the former limit, and the PEH of this section lying towards the latter limit. Each method has its role and does not exclude any other, because each increases the choice of tools we can bring to bear on a particular flow problem.

\section{Lattice Boltzmann methods}

\subsection{Historical development}

The lattice Boltzmann (LB) method is being increasingly used to simulate non-equilibrium flows, especially gas flows, at the micro- and nano-scale. Historically, the LB method was mainly developed as an alternative to solving the Navier-Stokes equations ${ }^{49,50,51}$, and it has been successfully applied to modelling complex multiphysical flows, including interfacial dynamics, flows in porous media, and suspensions. 
The underlying philosophy of the LB method is to design collision rules for computational particles (representing ensembles of real molecules) so that conservation laws for mass and momentum can be achieved. It developed out of lattice gas automata (LGA), which are discrete particle kinetic methods. LGA are designed to conserve mass and momentum by choosing collision laws, but another requirement is to have sufficient symmetry of the lattice in order to recover the Navier-Stokes equations ${ }^{52}$. Since macroscopic fluid dynamics is generally not sensitive to the details of intermolecular interactions, LGA are able to recover the correct Navier-Stokes behaviour.

In the LGA method, the particle occupation on numerical lattices can be described by a set of Boolean variables $n_{i}(\boldsymbol{x}, t), i=0,1 \ldots N$, where $N$ is the number of discrete velocities at each lattice node. The evolution equation for the LGA method is then very simple:

$$
n_{i}\left(\boldsymbol{x}+\boldsymbol{e}_{i}, t+1\right)=n_{i}(\boldsymbol{x}, t)+\Omega_{i},
$$

where $\boldsymbol{e}_{i}$ is the discrete velocity and $\Omega_{i}$ is the collision operator. The numerical procedure comprises two steps: streaming and collision. In the streaming step, every particle moves to the nearest node according to its discrete velocity. If two or more particles arrive at the same node, they collide and scatter according to a designated rule. Particle collisions do not obey Newton's Law microscopically, but a simple collision rule ensuring mass and momentum conservation.

Although it can recover Navier-Stokes behaviour in the continuum limit, the LGA model suffers from many drawbacks, including fluctuations in macroscopic properties and lack of Galilean invariance. Due to Boolean occupation, statistical noise is large so that an averaging process is required, which is computationally costly. To cure these shortcomings, McNamara and Zanetti ${ }^{53}$ proposed introducing a single particle distribution function to replace Boolean occupation, viz. $f_{i}=\left\langle n_{i}\right\rangle$. This idea was borrowed from the kinetic theory of gases, so that individual particle motion is absorbed in the distribution function, and the kinetic equation is then used to describe evolution of the particle distribution function. In this way, the fluctuations in macroscopic properties associated with the LGA method can be smoothed out. Note that although this idea was borrowed from the kinetic theory of gases, no formal theoretical link to this theory was established.

In the LB method, the evolution equation for the distribution function becomes:

$$
f_{i}\left(\boldsymbol{x}+\boldsymbol{e}_{i} \Delta \boldsymbol{x}, t+\Delta t\right)=f_{i}(\boldsymbol{x}, t)+\Omega_{i},
$$

where $\Delta \boldsymbol{x}$ is the lattice length and $\Delta t$ is the time step. Macroscopic properties such as density and velocity can then be obtained through:

$$
\rho=\sum_{i} f_{i}, \quad \rho \boldsymbol{u}=\sum_{i} f_{i} \boldsymbol{e}_{i} .
$$

When designing the collision operator, conservation of total mass and momentum needs to be satisfied. Therefore, the collision operator has to meet the following requirements:

$$
\sum_{i} \Omega_{i}=0, \quad \sum_{i} \Omega_{i} \boldsymbol{e}_{i}=0,
$$

and the simplest choice for a suitable collision operator is the BGK model ${ }^{54,55,56}$ :

$$
\Omega_{i}=-\frac{f_{i}-f_{i}^{e q}}{t_{c}},
$$

where $f_{i}^{e q}$ is the particle distribution function at equilibrium, and $t_{c}$ is the particle relaxation time.

Qian and colleagues ${ }^{55}$ provided a series of lattice models which they dubbed DnQm (with $n$ the dimension and $m$ the number of discrete velocities) with sufficient symmetry so that the lattice BGK 
method (outlined above) can recover the Navier-Stokes equations. Popular methods are D2Q9, D3Q15 and D3Q19; for example, the discrete velocities in a D2Q9 lattice method are:

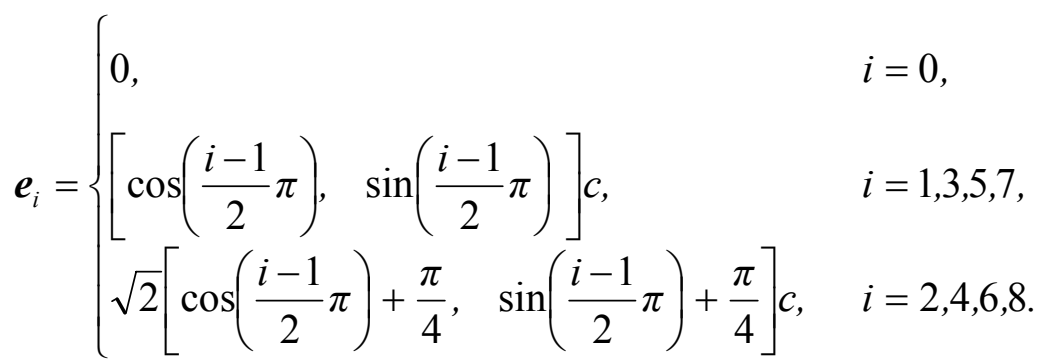

The equilibrium particle distribution function is

$$
\begin{gathered}
f_{i}^{e q}=\rho \omega_{i}\left[1+3 \boldsymbol{e}_{i} \cdot \boldsymbol{u}+\frac{9}{2}\left(\boldsymbol{e}_{i} \cdot \boldsymbol{u}\right)^{2}-\frac{3}{2} \boldsymbol{u}^{2}\right], \\
\text { with } \omega_{o}=\frac{4}{9} ; \quad \omega_{i}=\frac{1}{9}, i=1,3,5,7 ; \quad \omega_{i}=\frac{1}{36}, i=2,4,6,8 .
\end{gathered}
$$

These LB equations, and the ones that follow below, are generally expressed in lattice units, with $c=1$.

With the lattice BGK (LBGK) method, and the standardisation of the lattice models, the LB method started rapidly developing, and has now found application in complex fluid simulations, e.g. driven cavity flows ${ }^{57}$, turbulent flow ${ }^{58}$, and multiphase flows ${ }^{59,60,61}$.

\subsection{LB simulation of non-equilibrium flows}

The theoretical link between the LB method and the Boltzmann equation itself was established by $\mathrm{He}$ and $L \mathrm{OO}^{62}$, and $A \mathrm{Ab}^{63}$. This has led many researchers to investigate whether the LB method can be applied to rarefied gas dynamics, particularly because the LB boundary condition at solid surfaces only needs to provide the reflected particle distribution - it does not need to specify any macroscopic properties in the wall region. This is, as we have observed above, an important advantage over extended hydrodynamic models which struggle to find appropriate boundary conditions for macroscopic variables and their gradients ${ }^{20}$.

Although there is no theoretical proof that current models, such as LBGK, can capture high-order rarefaction effects, many researchers have been exploring the capability of LB methods numerically. One of the major hurdles in applying LB to non-equilibrium flow is imposing appropriate wall boundary conditions. Nie and co-workers ${ }^{64}$ used the LBGK method to simulate non-equilibrium gas flow using the 'bounce-back' wall boundary condition (in which particles colliding with the wall are reflected in the opposite direction). However, the bounce-back scheme is usually used for flows with no-slip-velocity at walls. Lim and colleagues ${ }^{65}$ used a combination of specular and bounce-back schemes to successfully capture some non-equilibrium effects, including velocity-slip at surfaces and the non-linear pressure gradient along a 2D channel.

Many other wall boundary conditions have also been proposed, including the virtual-wall collision scheme, described by Toschi and Succi ${ }^{66}$ as "a stochastic relaxation mechanism mimicking 'virtual' collisions between free-streaming particles and solid walls". Zhang and colleagues ${ }^{67}$ proposed a scheme that can incorporate the ubiquitous tangential accommodation coefficient; their argument was that the LB discrete velocity set is very limited, so the averaged reflection direction for a diffuse 
reflection should be normal to the surface. A tangential accommodation coefficient can then be used to characterize the reflection, which is usually between the diffuse and specular limits. Their simulation results were satisfactory in the slip flow regime and, surprisingly, the model also captures the 'Knudsen minimum' phenomenon.

However, probably the most significant slip-velocity model is the kinetic boundary condition proposed by Ansumali and Karlin ${ }^{68}$, based on the early work of Gatignol ${ }^{69}$. In this, the Maxwellian gas/surface interaction model is formulated in the discrete velocity scheme. Niu and co-workers ${ }^{70}$ proposed a similar kinetic boundary condition, and Tang and co-workers ${ }^{71}$ developed it further by incorporating an accommodation coefficient.

The standard LBGK method is a $2^{\text {nd }}$-order model (i.e. it retains $2^{\text {nd }}$-order terms in the expansion of the distribution function in Hermite polynomials), and has been successfully applied to simulate slip flows. But some researchers have claimed that the capabilities of LBGK reach beyond the slip flow regime $^{72}$; while $2^{\text {nd }}$-order LB methods can capture some high-order non-equilibrium phenomena, such as the tangential heat flux in an isothermal flow, they cannot capture the Knudsen layer flow which, as we outlined above, can often form a relatively large proportion of the flow domain ${ }^{73}$.

Since the Knudsen number is the usual indicator of non-equilibrium in gas flows, LB simulation results should be interpreted by $K n$ as well. However, $K n$ depends on how the molecular mean-free-path is calculated, which differs among models. This has caused some confusion in the LB literature. When comparing results between different methods, it is important to ensure the same definition of $K n$ is used; often a scaling coefficient has to be taken into account when considering results from different models ${ }^{67}$.

\subsection{Non-equilibrium LB methods}

Qian and Zhou ${ }^{74}$ attempted to prove that high-order hydrodynamics does exist in lattice-based models. However, sufficient symmetry for high-order moments cannot be achieved using available models. Since the standard LBGK method still behaves the same, more or less, as the Navier-Stokes equations, developing high-order LB methods for non-equilibrium flow has attracted significant recent interest.

Shan and colleagues ${ }^{75}$ proposed using the Hermite expansion to construct high-order LB models in which high-order moments, important for non-equilibrium gas flow, can be explicitly determined. Ansumali and co-workers ${ }^{76}$ demonstrated that, while such methods do improve the LB method's capabilities, the nonlinear constitutive relationship between stress and strain-rate in the Knudsen layer is still not well predicted. Figures 8 and 9 show a benchmark comparison of these proposed models with kinetic theory and DSMC data ${ }^{73}$.

Szalmás ${ }^{77}$ recently proposed a modification to implement the effect of high-order moments through a fast relaxation mode, which recalls the split of fast and slow moments proposed by Gorban and $\operatorname{Karlin}^{78}$. This method essentially uses two different relaxation times for low-order and high-order moments, and has been shown to capture Knudsen layer flow behaviour with an adjustable Knudsen layer thickness. In the same spirit as the regularized moment models outlined in section 2.1, Zhang and co-workers ${ }^{79}$, and $\mathrm{Niu}$ and co-workers ${ }^{80}$, added an additional term to their LB methods to account for omitted high-order moment effects. Although all these modifications have been shown 
to capture qualitatively the velocity profile in the Knudsen layer of a planar Couette flow, they are at an early stage and a quantitatively-accurate high-order LB method is still needed.

As we discussed in section 2.3, the proximity of a solid wall affects the free flight of gas molecules; in the Knudsen layer, gas molecules are more likely to collide with the wall rather than another gas molecule. The collisions between gas molecules and the wall will lead to an effective reduction of the mean-free-path of the gas, which should be taken into account in LB simulations. Implementation of this wall-effect in LB models can be found in the papers by Zhang and colleagues ${ }^{81,82}$, and Tang and colleagues $^{73}$, where the formula proposed by Lockerby and co-workers ${ }^{45}$ was used.

On implementing this local mean-free-path modification, the standard LBGK method is able to capture the Knudsen layer very well, as shown in figure $10 .{ }^{81}$ However, for a pressure-driven flow, as shown in figure 11, the discrepancy with kinetic theory predictions is still large, possibly because this modification may not be able to capture the high-order non-equilibrium effects caused by a pressure gradient. $^{81}$

Conversely, high-order LB methods fail to capture Knudsen layer characteristics in a Couette flow where no pressure gradient is present, but they have been shown to improve the prediction of pressure-driven flows ${ }^{73}$. Because the roots of the $4^{\text {th }}$-order Hermite polynomials are irrational, the discrete velocities cannot match lattice nodes. So additional effort, such as point-wise interpolation ${ }^{83}$, is therefore required and this dramatically increases the computational cost (because it then essentially becomes an off-lattice discrete velocity method for solving the kinetic Boltzmann equation). Worse still, these high-order LB methods with large numbers of discrete velocities are not numerically stable ${ }^{84}$. Chikatamarla and $\mathrm{Karlin}^{85}$ proposed a rational number approximation concept to avoid point-wise interpolation, and in a similar spirit a simplified high-order LB method has been proposed by Tang and colleagues ${ }^{73}$. In the latter case, for example, the 2D model is given by:

$$
\begin{gathered}
f_{i}^{e q}=\rho \omega_{i}\left(1+\frac{\boldsymbol{e}_{i} \cdot \boldsymbol{u}}{c_{s}^{2}}+\frac{\left(\boldsymbol{e}_{i} \cdot \boldsymbol{u}\right)^{2}}{2 c_{s}^{4}}-\frac{\boldsymbol{u} \cdot \boldsymbol{u}}{2 c_{s}^{2}}+\frac{\left(\boldsymbol{e}_{i} \cdot \boldsymbol{u}\right)^{3}}{2 c_{s}^{6}}-\frac{3 \boldsymbol{e}_{i} \cdot \boldsymbol{u}^{3}}{2 c_{s}^{4}}\right), \\
\omega_{o}=\frac{3}{8} ; \quad \omega_{i}=\frac{1}{12}, i=1-4 ; \quad \omega_{i}=\frac{1}{16}, i=5-8 ; \quad \omega_{i}=\frac{1}{96}, i=9-12,
\end{gathered}
$$

where $c_{s}=\sqrt{R T}$. The lattice velocities are:

$$
\begin{aligned}
& \boldsymbol{e}_{0}=0 \text {, } \\
& \boldsymbol{e}_{i}=\left[\cos \left(\frac{(i-1) \pi}{2}\right), \sin \left(\frac{(i-1) \pi}{2}\right)\right] c, \quad i=1-4, \\
& \boldsymbol{e}_{i}=\left[\cos \left(\frac{(i-5) \pi}{2}+\frac{\pi}{4}\right), \sin \left(\frac{(i-5) \pi}{2}+\frac{\pi}{4}\right)\right] \sqrt{2 c}, \quad i=5-8 \\
& \boldsymbol{e}_{i}=\left[\cos \left(\frac{(i-1) \pi}{2}\right), \sin \left(\frac{(i-1) \pi}{2}\right)\right] 2 c, \quad i=9-12,
\end{aligned}
$$

where $c=\sqrt{2 R T}$.

With the local mean-free-path modification also implemented, figures 10 and 11 show the method of Tang and colleagues ${ }^{73}$ not only captures the nonlinear constitutive behaviour of the stress and heatflux but also the high-order rarefaction effect in pressure-driven flows. Considering the influence of bounding surfaces on the local mean-free-path seems to be essential if the Knudsen layer flow is to be captured, as shown for the planar Couette case where no pressure gradient is present. In the 
pressure-driven flow, even with the wall effect incorporated, D2Q13 performs much better than D2Q9. This indicates that a high-order model is likely to have improved predictive capabilities for high-order rarefaction effects due to external forces, although capturing Knudsen layer effects still depends on a local mean-free-path modification.

For realistic non-equilibrium flows, Knudsen layer interference (or 'overlap') also has to be considered. A simple summative model has been used for flows between two parallel plates ${ }^{81,82,73}$, but this is difficult to extend to more complex geometries. The modified mean-free-path approach of Guo and co-workers ${ }^{36}$, mentioned in section 2.3 in connection with hydrodynamic models, has therefore now been implemented in the LB method ${ }^{86}$.

In order to capture physics beyond the NSF equations for non-equilibrium thermal flows, $5^{\text {th }}$-order velocity terms in the Hermite expansion to the equilibrium distribution function should be retained. This leads to a large number of discrete velocities. A non-equilibrium thermal model has been proposed by Zhang and co-workers ${ }^{82}$ to separate the different relaxation rates for momentum and energy by adopting a different distribution function for the energy density - as used by $\mathrm{He}$ and coworkers ${ }^{87}$ for no-velocity-slip and no-temperature-jump hydrodynamics - to describe the evolution of the energy. Only up to $3^{\text {rd }}$-order moments for the energy density equilibrium distribution function are then sufficient to describe the heat-flux beyond the NSF level. With, again, modification of the local mean-free-path in the wall region, the method can capture the velocity-slip and temperaturejump at the wall, in addition to the Knudsen layer flow characteristics.

\section{Conclusions}

Recent years have seen resurging international interest in methods for simulating rarefied and other non-equilibrium flows. This interest has been stimulated by the need for effective design tools for micro- and nano-scale systems, coupled with a growing appreciation that the fluid dynamics of these small-scale systems is often crucially different to that at the macro-scale.

Any review of this diverse field needs to be selective, and can provide only a snapshot of the techniques being explored. In this short article we have attempted to pick out some of the most important and exciting recent developments, and point to a few of the interesting links between them. We therefore conclude with some observations concerning the methods and models we have reviewed:

- Considering the hydrodynamic framework, the models now being pursued range from accurate but complex R13 and R20 moment models, to elegantly simple 'engineering models' of near-wall constitutive scaling (PEH). Each model strikes a different balance between simulation accuracy and computability, and choosing which one to use depends on the flow situation under investigation and the modelling requirements.

- Wall boundary conditions for extended hydrodynamic models are becoming increasingly wellunderstood, with new conditions now emerging that describe the micro-processes at the surface more realistically and which can capture non-equilibrium near-surface phenomena such as Knudsen layers.

- Translating the novel concept of near-wall constitutive scaling (in the form of 'effective meanfree-path' models) from hydrodynamics across to lattice Boltzmann methods is showing a surprising ability to predict some important rarefaction behaviour. Without this modification, 
however, high-order LB methods still fail to predict Knudsen layer flows, even if the prediction of rarefaction effects caused by external forces is improved.

- Simplified high-order LB methods incorporating near-wall scaling of the gas molecular mean-freepath offer a great deal of promise as simulation tools, with both computational efficiency and numerical accuracy, for a wide range of non-equilibrium flows in micro- and nano-scale systems.

\section{Acknowledgements}

The authors would like to thank Zhaoli Guo (Huazhong University of Science and Technology), Simon Mizzi (Rolls Royce plc), David Emerson, Guihua Tang, Xiao-Jun Gu and Robert Barber (all of Daresbury Laboratory), Duncan Lockerby (Warwick University) and Erik Arlemark (Strathclyde University) for useful discussions. This work is funded in the UK by the Engineering and Physical Sciences Research Council through grants EP/F002467/1, EP/D07455X/1, EP/D007488/1 and EP/F028865/1.

\section{References}

1. M Gad-el-Hak (1999) The fluid mechanics of microdevices. J. Fluids Eng.-Trans. ASME, 121:5-33

2. GE Karniadakis, A Beskok, N Aluru (2005) Microflows and nanoflows: fundamentals and simulation. Springer, New York

3. EB Arkilic, MA Schmidt, KS Breuer (1997) Gaseous slip flow in long microchannels. J. MEMS, 6:167-178

4. DA Lockerby, JM Reese, DR Emerson, RW Barber (2004) Velocity boundary condition at solid walls in rarefied gas calculations. Phys. Rev. E, 70:017303

5. M Knudsen (1909) Die gesetze der molecularströmung und der inner reibungsströmung der gase durch röhren. Annal. Physik, 28:75

6. SE Vargo, EP Muntz, GR Shiflett, WC Tang (1999) Knudsen compressor as a micro- and macroscale vacuum pump without moving parts or fluids. J. Vacuum Sci. Tech. A: Vacuum, Surface, Films, 17:2308

7. RK Agarwal, KY Yun, R Balakrishnan (2001) Beyond Navier-Stokes: Burnett equations for flows in the continuum-transition regime. Phys. Fluids, 13:3061-3085

8. F Sharipov, LMG Cumin, D Kalempa (2004) Plane Couette flow of binary gaseous mixture in the whole range of the Knudsen number. Eur. J. Mech. B-Fluids, 23:899

9. M Kalweit, D Drikakis (2008) Multiscale methods for micro/nano flows and materials. J. Comput. Theor. Nanosci., 5:1923

10. S Chapman, TG Cowling (1970) The mathematical theory of non-uniform gases. Cambridge University Press, UK

11. DA Lockerby, JM Reese, MA Gallis (2005) The usefulness of higher-order constitutive relations for describing the Knudsen layer. Phys. Fluids, 17:100609

12. DA Lockerby, JM Reese (2008) On the modelling of isothermal gas flows at the microscale. J. Fluid Mech., 604:235-261

13. H Struchtrup (2005) Macroscopic transport equations for rarefied gas flows. Springer

14. DA Lockerby, JM Reese (2003) High-resolution Burnett simulations of micro Couette flow and heat transfer. J. Comput. Phys., 188:333-347

15. H Grad (1949) On the kinetic theory of rarefied gases. Comm. Pure Appl. Math., 2:331-407

16. H Struchtrup, M Torrilhon (2003) Regularization of Grad's 13-moment equations: derivation and linear analysis. Phys. Fluids, 15:2668-2680 
17. H Struchtrup, M Torrilhon (2007) H theorem, regularization, and boundary conditions for linearized 13 moment equations. Phys. Rev. Lett., 99:014502

18. S Mizzi (2008) Extended macroscopic models for rarefied gas dynamics in micro-sized domains. $\mathrm{PhD}$ thesis, University of Strathclyde, UK

19. S Mizzi, XJ Gu, DR Emerson, RW Barber, JM Reese (2008) Computational framework for the regularized 20-moment equations for non-equilibrium gas flows. Int. J. Num. Meth. Fluids, 56:1433-1439

20. XJ Gu, DR Emerson (2007) A computational strategy for the regularized 13 moment equations with enhanced wall-boundary conditions. J. Comput. Phys., 225:263.

21. S Mizzi, DR Emerson, SK Stefanov, RW Barber, JM Reese (2007) Effects of rarefaction on cavity flow in the slip regime. J. Comput. Theoret. Nanosci., 4:817-822

22. L O'Hare, TJ Scanlon, DR Emerson, JM Reese (2008) Evaluating constitutive scaling models for application to compressible microflows. Int. J. Heat Mass Transf., 51:1281-1292

23. RS Myong, JM Reese, RW Barber, DR Emerson (2005) Velocity slip in microscale cylindrical Couette flow: the Langmuir model. Phys. Fluids, 17:087105

24. RS Myong, DA Lockerby, JM Reese (2006) The effect of gaseous slip on microscale heat transfer: an extended Graetz problem. Int. J. Heat Mass Transf., 49:2502-2513

25. JC Maxwell (1879) On stresses in rarified gases arising from inequalities of temperature. Phil. Trans. Roy. Soc. London, 170:231-256

26. R Schamberg (1947) The fundamental differential equations and the boundary conditions for high speed slip-flow, and their application to several specific problems. PhD thesis, California Institute of Technology, USA

27. C Cercignani (1969) Mathematical methods in kinetic theory. Plenum, New York

28. RG Deissler (1964) An analysis of second-order slip flow and temperature-jump boundary conditions for rarefied gases. Int. J. Heat Mass Transf., 7:681-694

29. AK Sreekanth (1969) Slip flow through long circular tubes, in: Rarefied Gas Dynamics, $6^{\text {th }}$ Symposium, Vol. 1. Academic, New York

30. YT Hsia, GA Domoto (1983) An experimental investigation of molecular rarefaction effects in gas lubricated bearings at ultra-low clearances. J. Lubrication Technol., 105:120-130

31. NG Hadjiconstantinou (2003) Comment on Cercignani's second-order slip coefficient. Phys Fluids, 15:2352-2354

32. J Maurer, P Tabeling, P Joseph, H Willaime (2003) Second-order slip laws in microchannels for helium and nitrogen. Phys. Fluids, 15:2613-2621

33. T Ewart, P Perrier, I Graur, JG Méolans (2006) Mass flow rate measurements in gas micro flows. Expts. Fluids, 41:487-498

34. Y Sone (1972) Flow induced by thermal-stress in rarefied-gas. Phys. Fluids, 15:1418-1423

35. DW Stops (1970) The mean free path of gas molecules in the transition regime. J. Phys. D: Appl. Phys., 3:685-696

36. ZL Guo, BC Shi, CG Zheng (2007) An extended Navier-Stokes formulation for gas flows in the Knudsen layer near a wall. EPL, 80:24001

37. M Fichman, G Hetsroni (2005) Viscosity and slip velocity in gas flow in microchannels. Phys. Fluids, 17:123102

38. JM Li, BX Wang, XF Peng (2000) 'Wall-adjacent layer' analysis for developed-flow laminar heat transfer of gases in microchannels. Int. J. Heat Mass Transf., 43:839-847

39. YH Sun, WK Chan, NY Liu (2003) A slip model for gas lubrication based on an effective viscosity concept. Proc. Inst. Mech. Engin., J-J. Eng. Tribology, 217:187-195 
40. G Schrag, G Wachutka (2002) Physically based modelling of squeeze film damping by mixed-level system simulation. Sensors \& Actuators A, 97-98:193

41. T Veijola. H Kuisma, J Lahdenperä, T Ryhänen (1995) Equivalent-circuit model of the squeezed gas film in a silicon accelerometer. Sensors \& Actuators A, 48:239

42. S Mizzi, RW Barber, DR Emerson, JM Reese, SK Stefanov (2007) A phenomenological and extended continuum approach for modelling non-equilibrium flows. Cont. Mech. Thermodyn., 19:273-283

43. L O'Hare, DA Lockerby, JM Reese, DR Emerson (2007) Near-wall effects in rarefied gas microflows: some modern hydrodynamic approaches. Int. J. Heat Fluid Flow, 28:37-43

44. JM Reese, Y Zheng, DA Lockerby (2007) Computing the near-wall region in gas micro- and nanofluidics: critical Knudsen layer phenomena. J. Comput. Theoret. Nanosci., 4:807-813

45. DA Lockerby, JM Reese, MA Gallis (2005) Capturing the Knudsen layer in continuum-fluid models of non-equilibrium gas flows. AIAA J., 43:1391-1393

46. CR Lilley, JE Sader (2007) Velocity gradient singularity and structure of the velocity profile in the Knudsen layer according to the Boltzmann equation. Phys. Rev. E, 76:026315

47. CR Lilley, JE Sader (2008) Velocity profile in the Knudsen layer according to the Boltzmann equation. Proc. Roy. Soc. A, 464:2015-2035

48. AJ Lofthouse, LC Scalabrin, ID Boyd (2008) Velocity slip and temperature jump in hypersonic aerothermodynamics. J. Thermophys. Heat Transf., 22:38-49

49. S Chen, GD Doolen (1998) Lattice Boltzmann method for fluid flows. Annu. Rev. Fluid Mech., 30:329-364

50. S Succi (2001) The lattice Boltzmann equation for fluid dynamics and beyond. Clarendon Press, Oxford

51. RR Nourgaliev, TN Dinh, TG Theofanous, D Joseph (2003) The lattice Boltzmann equation method: theoretical interpretation, numerics and implications. Int. J. Multiphase Flow, 29:117

52. U Frisch, B Hasslacher, Y Pomeau (1986) Lattice gas automata for the Navier-Stokes equations. Phys. Rev. Lett., 56:1505-1508

53. GR McNamara, G Zanetti (1988) Use of the Boltzmann equation to simulate lattice-gas automata. Phys. Rev. Lett., 61:2332

54. YH Qian (1990) Lattice gas and lattice kinetic theory applied to the Navier-Stokes equations. PhD thesis, Université Pierre et Marie Curie, Paris, France

55. YH Qian, D d'Humières, P Lallemand (1992) Lattice BGK Models for Navier-Stokes equation. Europhys. Lett., 17:479

56. S Chen, HD Chen, D Martinez, W Matthaeus (1991) Lattice Boltzmann model for simulation of magnetohydrodynamics. Phys. Rev. Lett., 67:3776-3779

57. S Hou, Q Zou, S Chen, G Doolen, AC Cogley (1995) Simulation of cavity flow by the lattice Boltzmann method. J. Comput. Phys., 118:329

58. DO Martinez, WH Mattaeus, S Chen, DC Montgomery (1994) Comparison of spectral method and lattice Boltzmann simulations of two-dimensional hydrodynamics. Phys. Fluids, 6:1285

59. XW Shan, HD Chen (1993) Lattice Boltzmann model for simulating flows with multiple phases and components. Phys. Rev. E, 47:1815

60. A Gunstensen, DH Rothman, S Zaleski, G Zanetti (1991) A lattice Boltzmann model of immiscible fluids. Phys. Rev. A, 43:4320

61. MR Swift, WR Osborn, JM Yeomans (1995) Lattice Boltzmann simulation of nonideal fluids. Phys. Rev. Lett., 75:830

62. X He, LS Luo (1997) A priori derivation of the lattice Boltzmann equation. Phys. Rev. E, 55:6333 
63. T Abe (1997) Derivation of the lattice Boltzmann method by means of the discrete ordinate method for the Boltzmann equation. J. Comp. Phys., 131:241-246

64. X Nie, GD Doolen, S Chen (2002) Lattice-Boltzmann simulations of fluidflows in MEMS. J. Stat. Phys., 107:279

65. CY Lim, C Shu, XD Niu, YT Chew (2002) Application of lattice Boltzmann method to simulate microchannel flows. Phys. Fluids, 14:2299

66. F Toschi, S Succi (2005) Lattice Boltzmann simulation at finite Knudsen numbers. Europhys. Lett., 69:549

67. YH Zhang, RS Qin, DR Emerson (2005) Lattice Boltzmann simulation of rarefied gas flows in microchannels. Phys. Rev. E, 71:047702

68. S Ansumali, IV Karlin (2002) Kinetic boundary conditions in the lattice Boltzmann method. Phys. Rev. E, 66:026311

69. R Gatignol (1977) Kinetic-theory boundary-conditions for discrete velocity gases. Phys. Fluids, 20:2022-2030

70. XD Niu, C Shu, YT Chew (2004) A lattice Boltzmann BGK model for simulation of micro flows. Europhys. Lett., 67:600

71. GH Tang, WQ Tao, YL He (2005) Lattice Boltzmann method for gaseous microflows using kinetic theory boundary conditions. Phys. Fluids, 17:058101

72. M Sbragaglia, S Succi (2006) Note on the lattice Boltzmann method beyond the ChapmanEnskog limits. Europhys. Lett., 73:370

73. GH Tang, YH Zhang, DR Emerson (2008) Lattice Boltzmann models for nonequilibrium gas flows. Phys. Rev. E, 77:046701

74. YH Qian, Y Zou (2000) Higher-order dynamics in lattice-based models using the Chapman-Enskog method. Phys. Rev. E, 61:2103

75. XW Shan, XF Yuan, HD Chen (2006) Kinetic theory representation of hydrodynamics: a way beyond the Navier-Stokes equation. J. Fluid Mech., 550:413

76. S Ansumali, IV Karlin, S Arcidiacono, A Abbas, NI Prasianakis (2007) Hydrodynamics beyond Navier-Stokes: exact solution to the lattice Boltzmann hierarchy. Phys. Rev. Lett., 98:124502

77. L Szalmás (2007) Knudsen layer theory for high-order lattice Boltzmann models. EPL, 80:24003

78. AN Gorban, IV Karlin (2006) Quasi-equilibrium closure hierarchies for the Boltzmann equation. Physica A, 360:325-364

79. R Zhang, X Shan, H Chen (2006) Efficient kinetic method for fluid simulation beyond the NavierStokes equation. Phys. Rev. E, 74:046703

80. XD Niu, SA Hyodo, T Munekata, K Suga (2007) Kinetic lattice Boltzmann method for microscale gas flows: issues on boundary condition, relaxation time, and regularization. Phys. Rev. E, 76:036711

81. YH Zhang, XJ Gu, RW Barber, DR Emerson (2006) Capturing Knudsen layer phenomena using a lattice Boltzmann model. Phys. Rev. E, 74:046704

82. YH Zhang, XJ Gu, RW Barber, DR Emerson (2007) Modelling thermal flow in the transition regime using a lattice Boltzmann approach. EPL, 77:30003

83. XY He, LS Luo, M Dembo (1996) Some progress in lattice Boltzmann method, 1: Nonuniform mesh grids. J. Comput. Phys., 129:357-363

84. S Succi, IV Karlin, H Chen (2002) Colloquium: Role of the $H$ theorem in lattice Boltzmann hydrodynamic simulations. Rev. Modern Phys., 74:1203

85. SS Chikatamarla, IV Karlin (2006) Entropy and Galilean invariance of lattice Boltzmann theories. Phys. Rev. Lett., 97:190601 
86. GH Tang, YH Zhang, XJ Gu, DR Emerson (2008) Lattice Boltzmann modelling Knudsen layer effect in non-equilibrium flows. J. Phys. $A$ (under consideration)

87. X He, S Chen, GD Doolen (1998) A novel thermal model for the lattice Boltzmann method in incompressible limit. J. Comput. Phys., 146:282 


\begin{tabular}{|c|c|c|c|c|}
\hline Reference & Study & Gas molecule & $A_{1}$ & $A_{2}$ \\
\hline Maxwell $(1879)^{25}$ & theoretical & $\begin{array}{l}\text { monatomic } \\
\text { \& diatomic }\end{array}$ & 1.0 & 0.0 \\
\hline Schamberg $(1947)^{26}$ & theoretical & monatomic & 1.0 & 1.31 \\
\hline Cercignani $(1969)^{27}$ & theoretical & monatomic & 1.15 & 0.98 \\
\hline Deissler $(1964)^{28}$ & theoretical & $\begin{array}{l}\text { monatomic } \\
\& \text { diatomic }\end{array}$ & 1.0 & 1.13 \\
\hline Sreekanth (1969) ${ }^{29}$ & experimental $^{*}$ & Nitrogen & 1.15 & 0.14 \\
\hline Hsia \& Domoto $(1983)^{30}$ & theoretical & $\begin{array}{l}\text { monatomic } \\
\& \text { diatomic }\end{array}$ & 1.0 & 0.5 \\
\hline Karniadakis et al. (2005) & theoretical & $\begin{array}{l}\text { monatomic } \\
\text { \& diatomic }\end{array}$ & 1.0 & -0.5 \\
\hline Hadjiconstantinou (2003) & theoretical & monatomic & 1.11 & 0.61 \\
\hline Maurer et al. $(2003)^{32}$ & experimental & $\begin{array}{c}\text { Nitrogen } \\
\text { Helium }\end{array}$ & $\begin{array}{l}1.3 \pm 0.05 \\
1.2 \pm 0.05\end{array}$ & $\begin{array}{l}0.26 \pm 0.1 \\
0.23 \pm 0.1\end{array}$ \\
\hline Ewart et al. $(2006)^{33}$ & experimental $^{*}$ & Nitrogen & $1.32 \pm 0.1$ & $0.32 \pm 0.08$ \\
\hline Maxwell-Burnett $(2004)^{4}$ & theoretical & $\begin{array}{c}\text { monatomic } \\
\text { diatomic }\end{array}$ & $\begin{array}{l}1.0 \\
1.0\end{array}$ & $\begin{array}{l}0.19 \\
0.145\end{array}$ \\
\hline
\end{tabular}

* Data for cylindrical Poiseuille flow.

Table 1. List of proposed $1^{\text {st }}$ - and $2^{\text {nd }}$-order coefficients, $A_{1}$ and $A_{2}$ respectively, in the slip-velocity condition, equation (5); reported in the literature for a diffuse solid boundary. For comparison purposes, all values have been re-normalised to a common mean-free-path definition: $\lambda=\mu \sqrt{\pi / 2 p \rho}$, where $\mu$ is the gas viscosity, $p$ the pressure, and $\rho$ the density. 


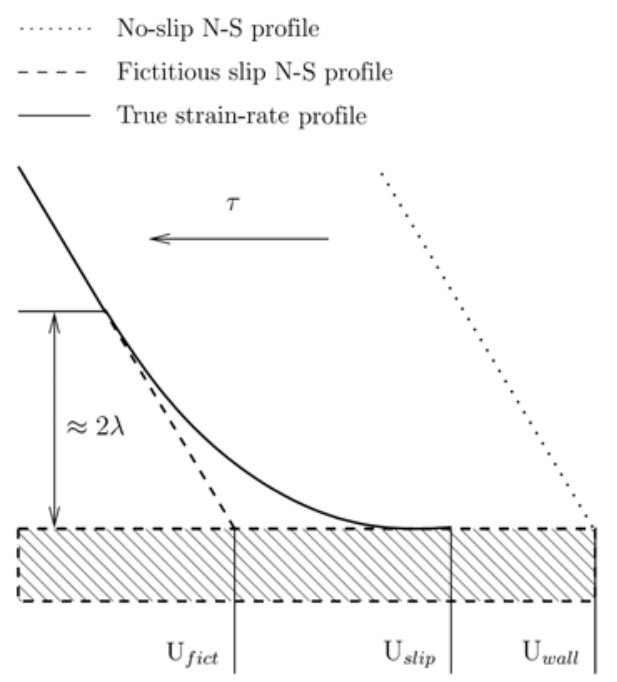

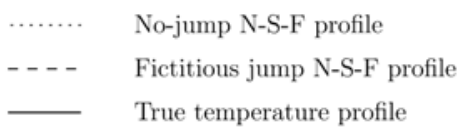



Figure 1. Schematics of the momentum and thermal Knudsen layers in Kramers' problem (left) and the temperature-jump problem (right), respectively. Kramers' flow is the incompressible, isothermal flow of a gas in a half-space under a constant shear stress applied tangentially to a stationary planar wall. The temperature-jump problem is a constant heat flux in a quiescent gas in a half-space, applied normally to a planar wall. Both problems produce linear velocity or temperature gradients, respectively, except in the Knudsen layers extending some $2 \lambda$ from the wall. Subscripts refer to: wall, values of the solid wall velocity/temperature; slip/jump, actual values of the gas slip-velocity and temperature-jump due to incomplete accommodation with the surface; fict, the 'fictitious' values of slip and jump that need to be imposed if the linear Navier-Stokes model is required to be accurate in the bulk flow beyond the Knudsen layer (but not within it). 


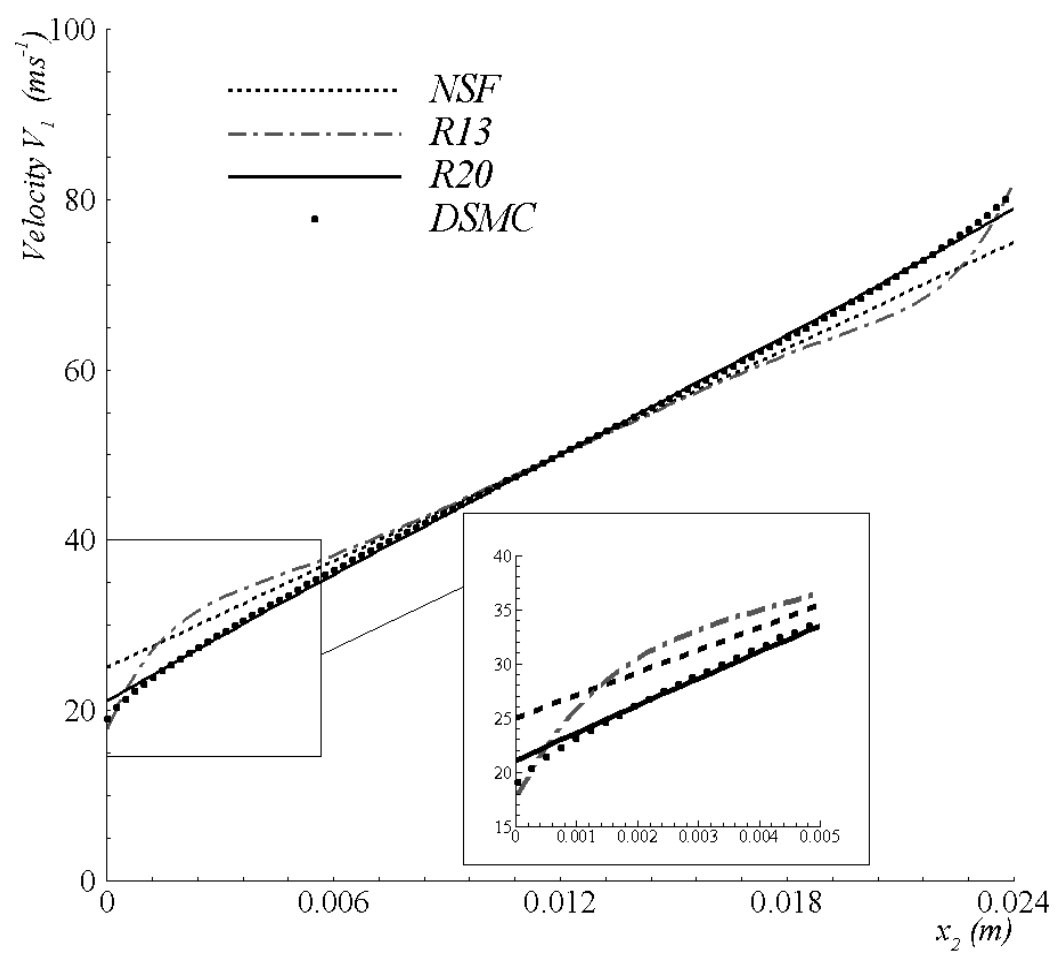

Figure 2. Planar Couette flow (Kn=0.5, argon gas), cross-channel dimensional velocity profiles, comparing the NSF slip solution to the R13 and R20 moment models and benchmark DSMC data. The channel surfaces are at $x_{2}=0 \mathrm{~m}$ (stationary wall) and $x_{2}=0.024 \mathrm{~m}$ (moving wall). Note that the NSF model is unable to capture the non-linear behaviour extending throughout the flow-field. The R13 model is excessively non-linear close to the wall (see insert magnification of near-wall solutions), but recovers most of the bulk flow behaviour. In this case, the R20 model manages to reproduce quite well both the non-linear behaviour close to the wall (the Knudsen layer), and the bulk flow. (Reprinted with permission from S Mizzi, Extended macroscopic models for rarefied gas dynamics in micro-sized domains, PhD thesis, University of Strathclyde, UK, 2008.) 


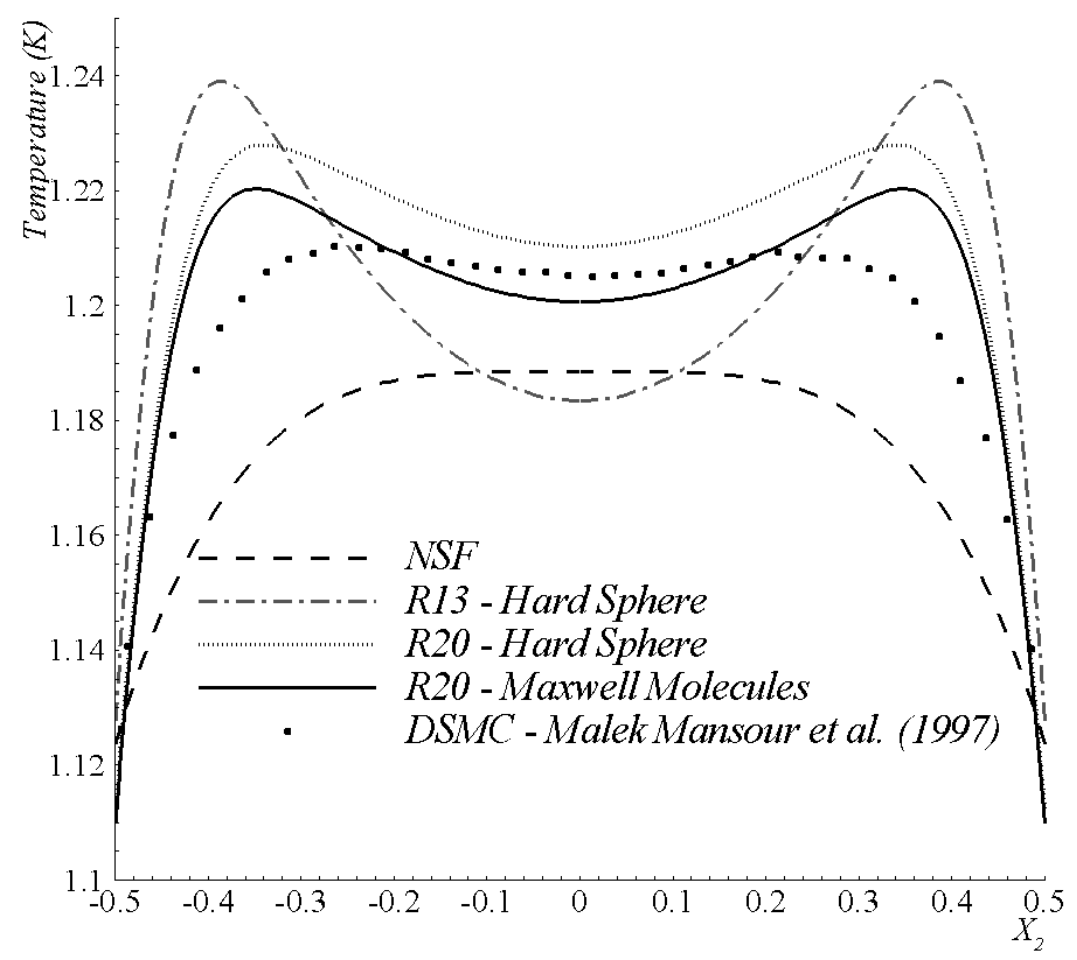

Figure 3. Force-driven planar Poiseuille flow $(K n=0.1)$, cross-channel temperature profiles, comparing various extended hydrodynamic solutions with benchmark DSMC (hard-sphere molecule) results. The bimodality of the temperature profile - not captured in any way by the conventional NSF model is qualitatively predicted by both the R13 and R20 models. Note that, although the collisional production term in the derivation of the R20 equations is for Maxwell-molecules only, using the R20 model together with a hard-sphere viscosity/temperature law can affect the results. (Reprinted with permission from S Mizzi, Extended macroscopic models for rarefied gas dynamics in micro-sized domains, PhD thesis, University of Strathclyde, UK, 2008.) 

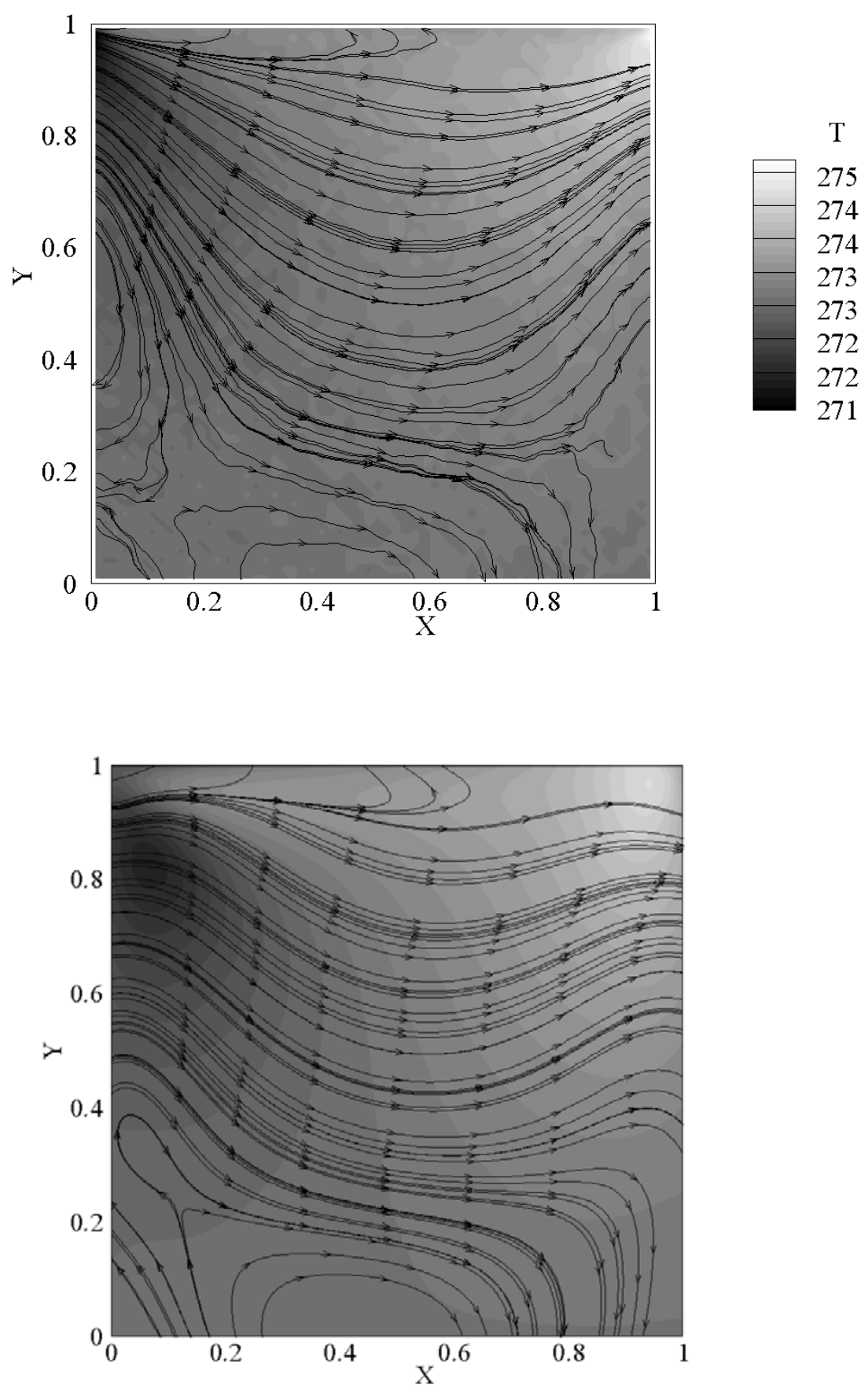

Figure 4. Lid-driven cavity flow in $2 \mathrm{D}(K n=0.1$, argon gas), heat flux stream-traces superimposed on a filled-contour plot of temperature (indicated, in degrees K); (top) results from a DSMC simulation, (bottom) results from the R20 model. Note the reversed heat-fluxes, i.e. in both solutions heat energy 'flows' from cold to hot regions. There is generally good agreement between the DSMC data and the R20 model results. (Reprinted with permission from S Mizzi, Extended macroscopic models for rarefied gas dynamics in micro-sized domains, PhD thesis, University of Strathclyde, UK, 2008.) 


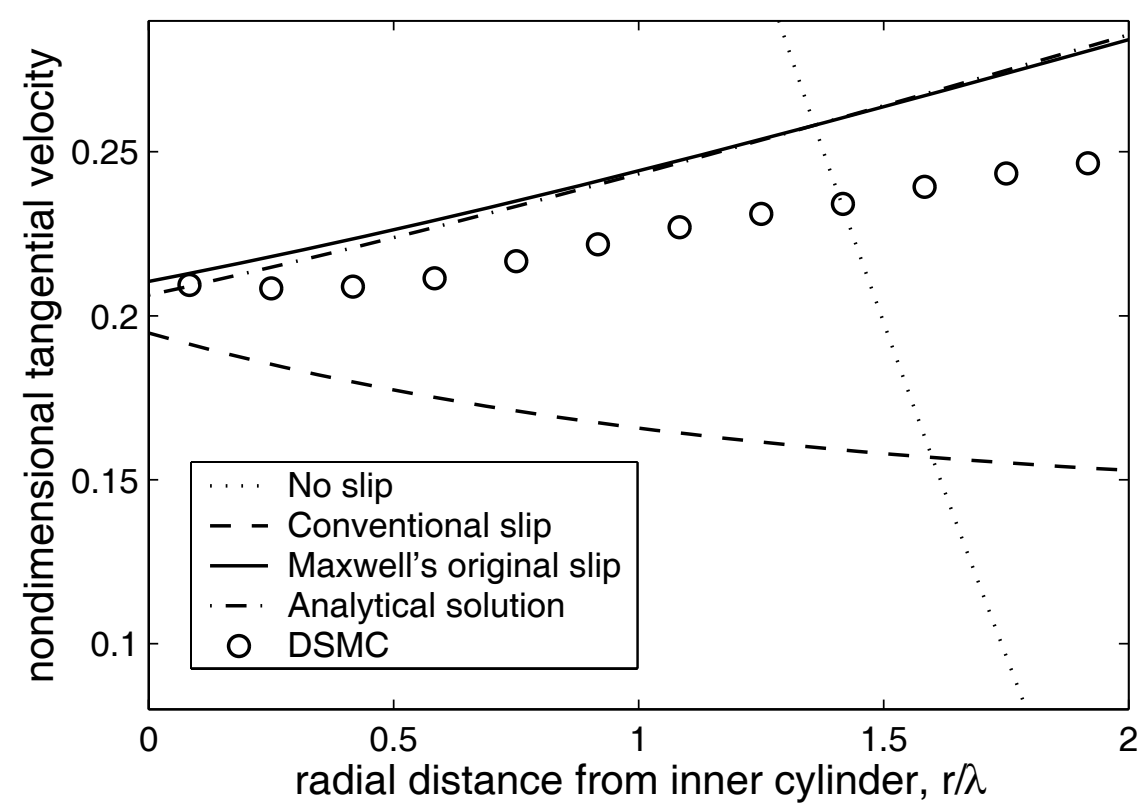

Figure 5. Cylindrical Couette flow ( $K n=0.5$, argon gas), non-dimensional velocity radial profiles, comparing hydrodynamic solutions calculated using various slip-velocity models with benchmark DSMC data. The inner wall (at $r / \lambda=0$ ) is rotating with a tangential velocity approximately a third the speed of sound relative to the outer wall (at $r / \lambda=2)$, the momentum accommodation coefficient is 0.1. Note the inverted velocity profile, captured by DSMC and an analytical kinetic theory but not by a hydrodynamic model (in this case, the NSF equations) unless the Maxwell original slip equation ${ }^{25}$ is implemented properly in this curved geometry. (Reprinted from DA Lockerby, JM Reese, DR Emerson, RW Barber (2004) Velocity boundary condition at solid walls in rarefied gas calculations. Phys. Rev. E, 70:017303.) 


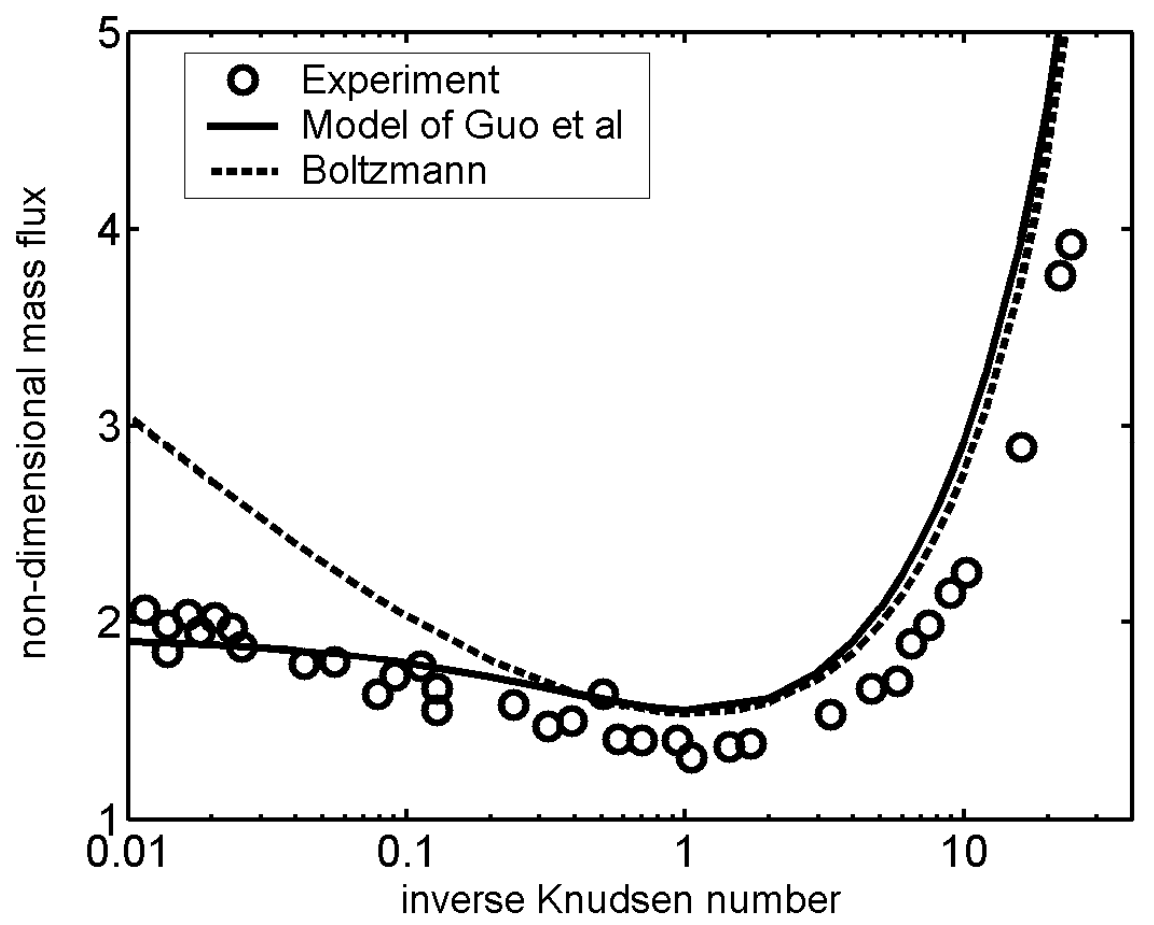

Figure 6. Planar Poiseuille flow at various (inverse) $K n$, non-dimensional mass flux, comparing predictions using the model of Guo and colleagues ${ }^{36}$ (with $2^{\text {nd }}$-order slip boundary conditions) with a solution of the Boltzmann equation and experimental data. While there is still some quantitative inexactness, the 'Knudsen minimum' (or 'Knudsen paradox') in the mass flux is clearly captured by the near-wall constitutive scaling model. (Reprinted with permission from ZL Guo, BC Shi, CG Zheng (2007) An extended Navier-Stokes formulation for gas flows in the Knudsen layer near a wall. EPL, 80:24001.) 


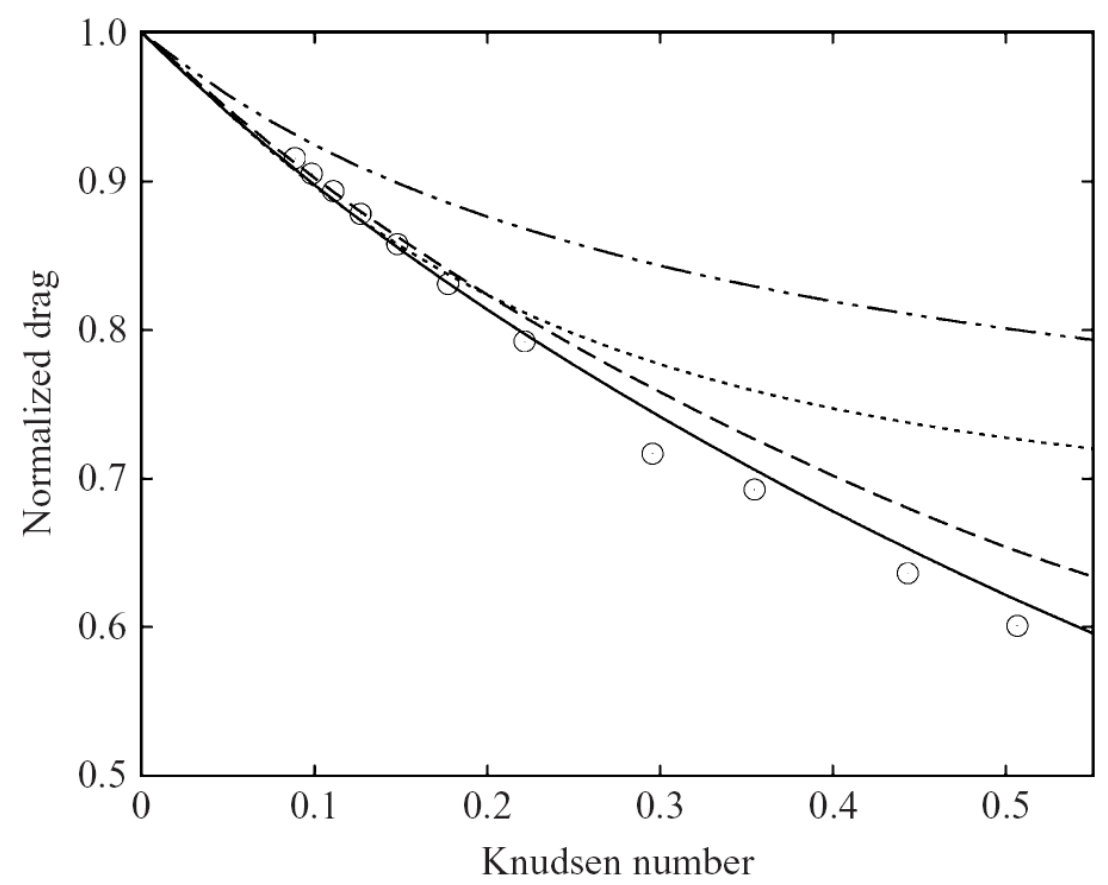

Figure 7. Flow around a sphere at various $K n$, normalized drag force, comparing predictions using the near-wall constitutive scaling model of Lockerby and Reese $(--)$ with Basset's classical slip solution $(-\cdots-)$, Cercignani's $2^{\text {nd }}$-order slip solution $(\cdots)$, BGK kinetic theory results $(0)$, and experimental data (-). (Reprinted with permission from DA Lockerby, JM Reese (2008) On the modelling of isothermal gas flows at the microscale. J. Fluid Mech., 604:235-261.) 


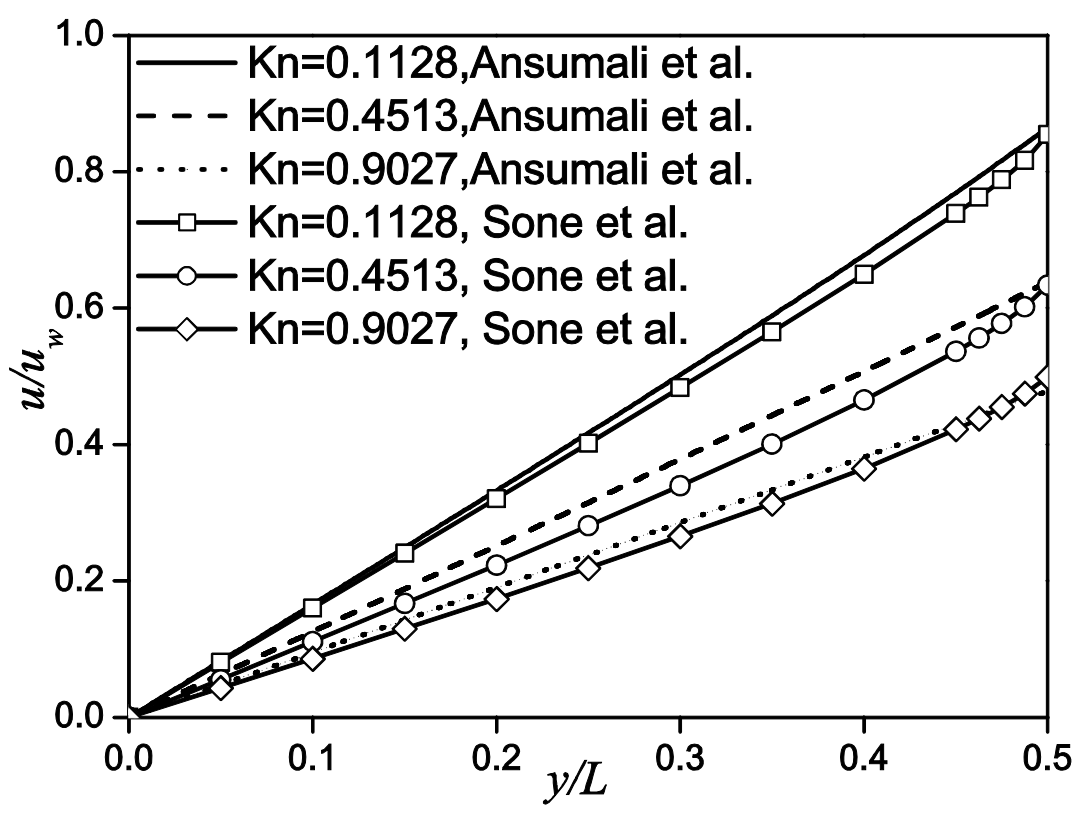

Figure 8. Planar Couette flow at various $K n$, cross-half-channel velocity profiles (nondimensionalised with the moving-surface speed of $u_{w}$ ), comparing analytical results from a high-order LB method ${ }^{76}$ with a solution of the linearised Boltzmann equation by Y Sone, S Takata, T Ohwada, Eur. J. Mech. BFluids, 9:273 (1990). (Reproduced from GH Tang, YH Zhang, DR Emerson (2008) Lattice Boltzmann models for nonequilibrium gas flows. Phys. Rev. E, 77:046701.) 


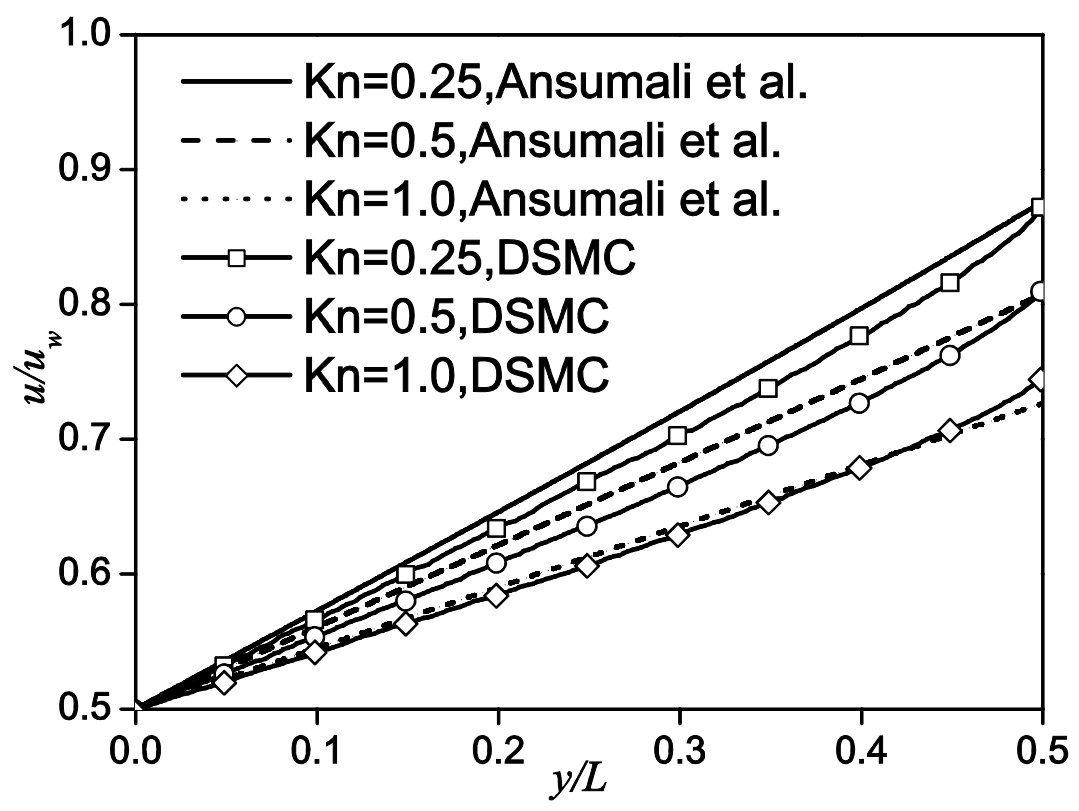

Figure 9. Planar Couette flow at various $K n$, cross-half-channel velocity profiles (nondimensionalised with the moving-surface speed of $u_{w}$ ), comparing analytical results from a high-order LB method ${ }^{76}$ with benchmark DSMC data. (Reproduced from GH Tang, YH Zhang, DR Emerson (2008) Lattice Boltzmann models for nonequilibrium gas flows. Phys. Rev. E, 77:046701.) 


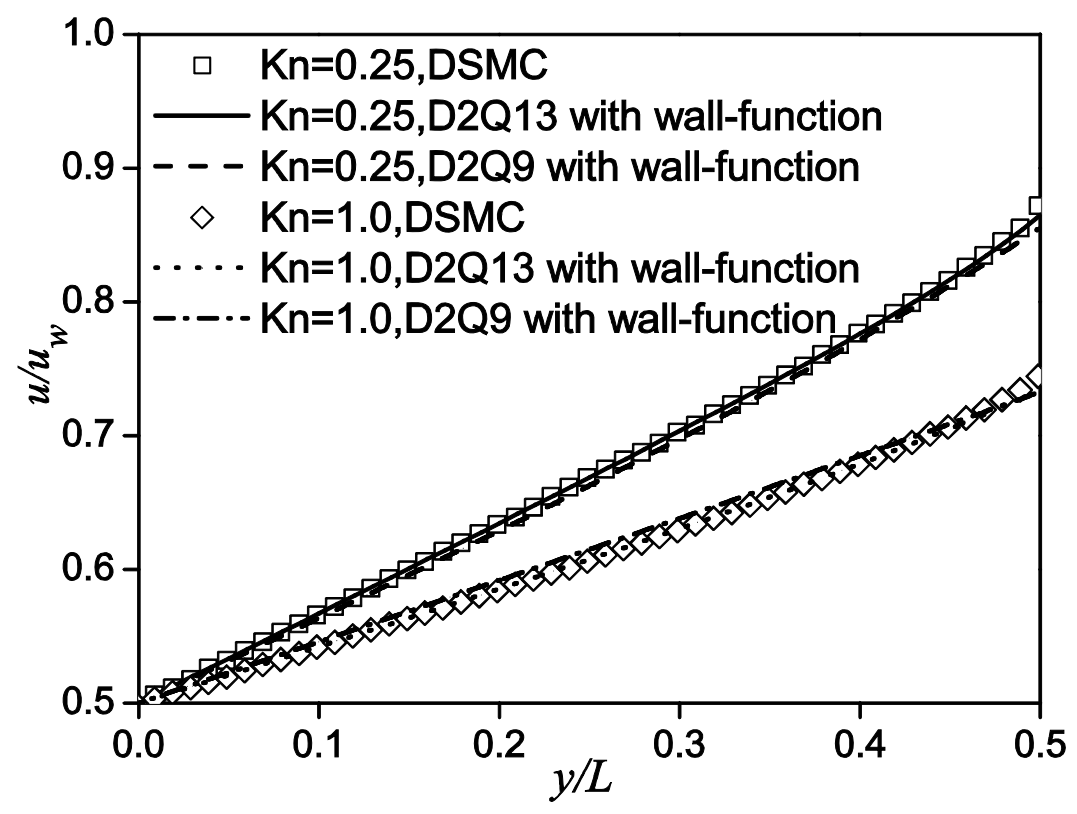

Figure 10. Planar Couette flow $(K n=0.25,1.0)$, cross-half-channel velocity profiles (nondimensionalised with the moving-surface speed of $u_{w}$ ), comparing D2Q9 and D2Q13 LB methods (including local mean-free-path modification, here labelled 'wall-function') with benchmark DSMC data. (Reproduced from GH Tang, YH Zhang, DR Emerson (2008) Lattice Boltzmann models for nonequilibrium gas flows. Phys. Rev. E, 77:046701.) 


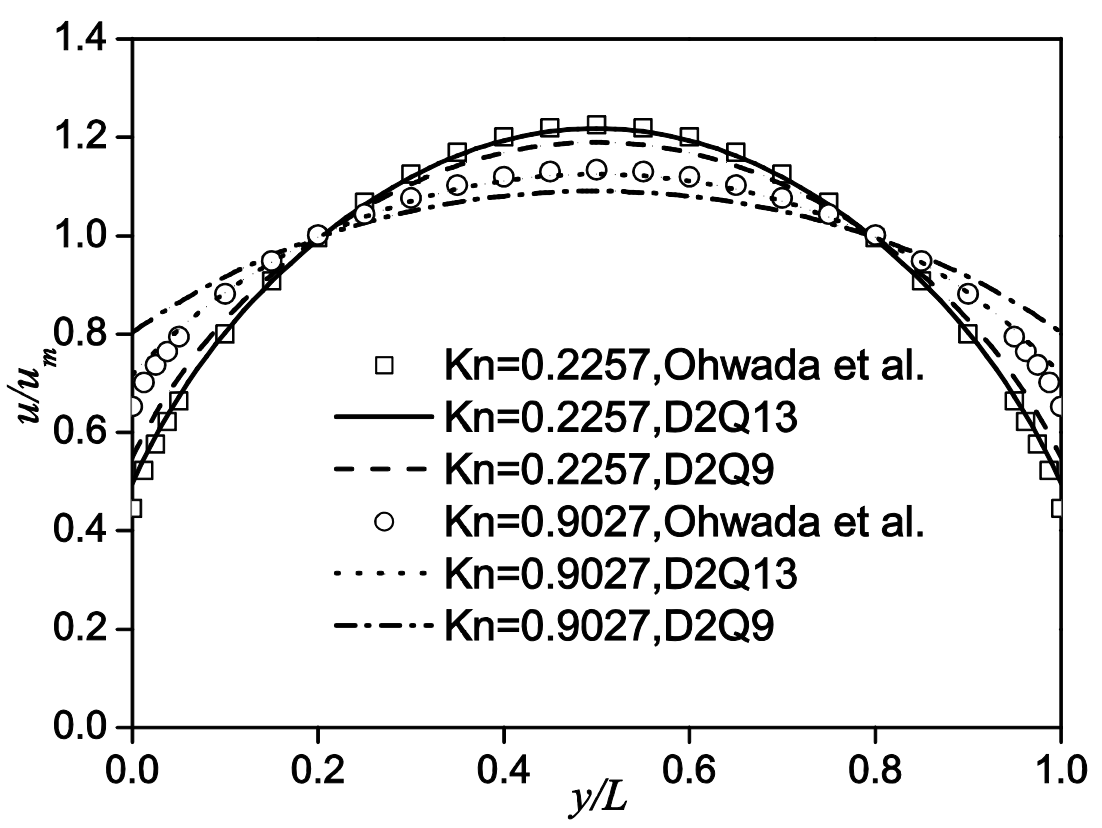

Figure 11. Planar Poiseuille flow $(K n=0.2257,0.9027)$, cross-channel velocity profiles (nondimensionalised with the average flow speed of $u_{m}$ ), comparing D2Q9 and D2Q13 LB methods (including local mean-free-path modification) with a solution of the linearised Boltzmann equation by T Ohwada, Y Sone, K Aoki, Phys. Fluids A, 1:1588 (1989). (Reproduced from GH Tang, YH Zhang, DR Emerson (2008) Lattice Boltzmann models for nonequilibrium gas flows. Phys. Rev. E, 77:046701.) 


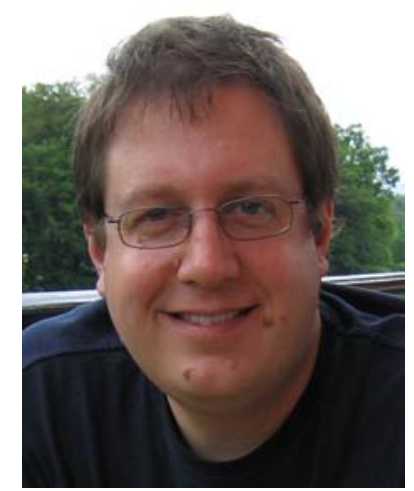

Born in London in 1967, Jason Reese graduated in Physics from Imperial College London, and completed his Masters and Doctoral research in Applied Mathematics at the University of Oxford in 1993. After postdoctoral work at the Technische Universitaet Berlin and the University of Cambridge, he became a Lecturer in Fluid Dynamics at the University of Aberdeen, and subsequently Lecturer and ExxonMobil Engineering Fellow at King's College London. In 2003 he was appointed the Weir Professor of Thermodynamics and Fluid Mechanics in the University of Strathclyde, Glasgow, Scotland. He is a Fellow of the Royal Society of Edinburgh, of the Institution of Mechanical Engineers, and of the Institute of Physics in the UK. His scientific interests focus on non-equilibrium fluid dynamics, in particular on modelling hypersonic aerodynamics and micro- and nano-scale flows.

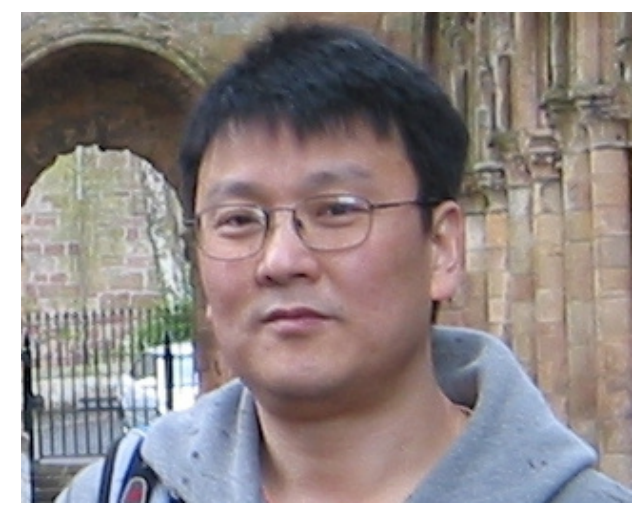

Yonghao Zhang obtained a first degree in Thermal Power Engineering from Jiangsu University of Technology and a Masters degree in Fluid Mechanics from East China University of Technology. After three years working in industry, he started his PhD research in Mechanical Engineering at the University of Aberdeen, UK. In 2001, he joined Daresbury Laboratory as a research scientist. Since 2007, he has been working at the University of Strathclyde as the John Anderson Research Lecturer in Mechanical Engineering. His research is mainly in microfluidics, microdroplet technologies and non-equilibrium gas dynamics. 